\title{
Radiogenic and Muon-Induced Backgrounds in the LUX Dark Matter Detector
}

D.S. Akerib ${ }^{b}$, H.M. Araújo ${ }^{d}$, X. Bai ${ }^{\text {h }}$, A.J. Bailey ${ }^{\text {d }}$,J. Balajthy ${ }^{\text {, E. Bernard }}$, A. Bernstein ${ }^{\mathrm{f}}$, A. Bradley $^{\mathrm{b}}$, D. Byram ${ }^{\mathrm{r}}$, S.B. Cahn ${ }^{\mathrm{s}}$, M.C. Carmona-Benitez ${ }^{\mathrm{b}, \mathrm{n}}$, C. Chan ${ }^{\mathrm{a}}$, J.J. Chapman ${ }^{\mathrm{a}}$, A.A. Chiller ${ }^{\mathrm{r}}$, C. Chiller ${ }^{\mathrm{r}}$, T. Coffey ${ }^{\mathrm{b}}$, A. Currie ${ }^{\mathrm{d}}$, L. de Viveiros ${ }^{\mathrm{g}}$, A. Dobi ${ }^{\mathrm{p}}$, J. Dobson ${ }^{\mathrm{o}}$, E. Druszkiewicz ${ }^{\mathrm{q}}$, B. Edwards ${ }^{\mathrm{s}}$, C.H. Faham ${ }^{\mathrm{a}, \mathrm{e}}$, S. Fiorucci ${ }^{\mathrm{a}}$, C. Flores ${ }^{\mathrm{m}}$, R.J. Gaitskell ${ }^{\text {a }}$, V.M. Gehman ${ }^{1}$, C. Ghag ${ }^{\mathrm{k}}$, K.R. Gibson ${ }^{\mathrm{b}}$, M.G.D. Gilchriese ${ }^{1}$, C. Hall ${ }^{\mathrm{P}}$, S.A. Hertel ${ }^{\mathrm{s}}$, M. Horn ${ }^{\mathrm{s}}$,

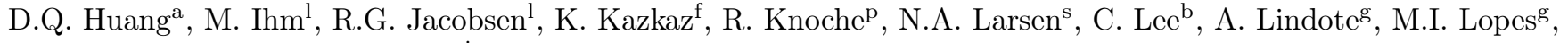
D.C. Malling ${ }^{\mathrm{a}, *}$, R. Mannino ${ }^{\mathrm{j}}$, D.N. McKinsey ${ }^{\mathrm{s}}$, D.-M. Mei ${ }^{\mathrm{r}}$, J. Mock ${ }^{\mathrm{m}}$, M. Moongweluwan ${ }^{\mathrm{q}}$, J. Morad ${ }^{\mathrm{m}}$, A.St.J. Murphy ${ }^{\mathrm{o}}$, C. Nehrkorn ${ }^{\mathrm{n}}$, H. Nelson ${ }^{\mathrm{n}}$, F. Neves ${ }^{\mathrm{g}}$, R.A. Ott ${ }^{\mathrm{m}}$, M. Pangilinan ${ }^{\mathrm{a}}$, P.D. Parker ${ }^{\mathrm{s}}$, E.K. Pease ${ }^{\mathrm{s}}$, K. Pech ${ }^{\mathrm{b}}$, P. Phelps ${ }^{\mathrm{b}}$, L. Reichhart ${ }^{\mathrm{k}}$, T. Shutt ${ }^{\mathrm{b}}$, C. Silva ${ }^{\mathrm{g}}$, V.N. Solovov ${ }^{\mathrm{g}}$, P. Sorensen ${ }^{\mathrm{f}}$, K. O'Sullivan ${ }^{\mathrm{s}}$, T.J. Sumner ${ }^{\mathrm{d}}$,

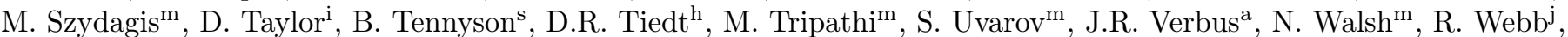
J.T. White ${ }^{\mathrm{j}}$, M.S. Witherell ${ }^{\mathrm{n}}$, F.L.H. Wolfs ${ }^{\mathrm{q}}$, M. Woods ${ }^{\mathrm{m}}$, C. Zhang ${ }^{\mathrm{r}}$

\author{
${ }^{a}$ Brown University, Dept. of Physics, 182 Hope St., Providence RI 02912, USA \\ ${ }^{b}$ Case Western Reserve University, Dept. of Physics, 10900 Euclid Ave, Cleveland OH 44106, USA \\ ${ }^{c}$ Harvard University, Dept. of Physics, 17 Oxford St., Cambridge MA 02138, USA \\ ${ }^{d}$ Imperial College London, High Energy Physics, Blackett Laboratory, London SW7 2BZ, UK \\ ${ }^{e}$ Lawrence Berkeley National Laboratory, 1 Cyclotron Rd., Berkeley CA 94720, USA \\ ${ }^{f}$ Lawrence Livermore National Laboratory, 7000 East Ave., Livermore CA 94550, USA \\ ${ }^{g}$ LIP-Coimbra, Department of Physics, University of Coimbra, Rua Larga, 3004-516 Coimbra, Portugal \\ ${ }^{h}$ South Dakota School of Mines and Technology, 501 East St Joseph St., Rapid City SD 57701, USA \\ ${ }^{i}$ South Dakota Science and Technology Authority, Sanford Underground Research Facility, Lead, SD 57r54, USA \\ ${ }^{j}$ Texas A \& $M$ University, Dept. of Physics, College Station TX 7r843, USA \\ ${ }^{k}$ University College London, Department of Physics and Astronomy, Gower Street, London WC1E 6BT, UK \\ ${ }^{l}$ University of California Berkeley, Department of Physics, Berkeley CA 94720, USA \\ ${ }^{m}$ University of California Davis, Dept. of Physics, One Shields Ave., Davis CA 95616, USA \\ ${ }^{n}$ University of California Santa Barbara, Dept. of Physics, Santa Barbara, CA, USA \\ ${ }^{\circ}$ University of Edinburgh, SUPA, School of Physics and Astronomy, Edinburgh, EH9 3JZ, UK \\ ${ }^{p}$ University of Maryland, Dept. of Physics, College Park MD 20742, USA \\ ${ }^{q}$ University of Rochester, Dept. of Physics and Astronomy, Rochester NY 14627, USA \\ ${ }^{r}$ University of South Dakota, Dept. of Physics, 414E Clark St., Vermillion SD 57069, USA \\ ${ }^{s}$ Yale University, Dept. of Physics, 217 Prospect St., New Haven CT 06511, USA
}

\section{Abstract}

The Large Underground Xenon (LUX) dark matter experiment aims to detect rare low-energy interactions from Weakly Interacting Massive Particles (WIMPs). The radiogenic backgrounds in the LUX detector have been measured and compared with Monte Carlo simulation. Measurements of LUX high-energy data have provided direct constraints on all background sources contributing to the background model. The expected background rate from the background model for the 85.3 day WIMP search run is $\left(2.6 \pm 0.2_{\text {stat }} \pm 0.4_{\text {Sys }}\right) \times 10^{-3}$ events $\mathrm{keV}_{e e}^{-1} \mathrm{~kg}^{-1} \mathrm{day}^{-1}$ in a $118 \mathrm{~kg}$ fiducial volume. The observed background rate is $\left(3.6 \pm 0.4_{\text {stat }}\right) \times 10^{-3}$ events $\mathrm{keV}_{e e}^{-1} \mathrm{~kg}^{-1}$ day $^{-1}$, consistent with model projections. The expectation for the radiogenic background in a subsequent one-year run is presented.

Keywords: LUX, dark matter, radioactive background, material screening, simulation

\section{Introduction}

The LUX experiment [1, 2] uses $370 \mathrm{~kg}$ of liquid Xe to search for nuclear recoil (NR) signatures from WIMP dark matter $[3,4,5]$. The LUX detector reconstructs event energy, position, and recoil type through its collection of scintillation (S1) and electroluminescence (S2) signals. LUX seeks sensitivity to rare WIMP interactions at energies on the order of several $\mathrm{keV}$. The extremely low WIMP in-

${ }^{*}$ Corresponding Author: David_Malling@brown.edu teraction rate necessitates precise control of background event rates in the detector.

A particle that produces a WIMP search background in LUX must mimic a WIMP signature in several ways. WIMPs are expected to interact with Xe nuclei in the active region, creating a NR event. WIMP interactions will be single-scatter ( $\mathrm{SS}$ ) events, distributed homogeneously in the active region. The LUX WIMP search energy window is defined in the range $3.4-25 \mathrm{keV}_{n r}$, where the "nr" subscript denotes that the energy was deposited by a nuclear recoil [2]. This window captures $80 \%$ of all WIMP interactions, assuming a WIMP mass of $100 \mathrm{GeV}$ and stan- 
dard galactic dark matter halo parameters as described in $2]$.

The dominant background in the LUX WIMP search, which principally constrains the experimental sensitivity published for the 85.3 day run [2], is low-energy electron recoil (ER) signatures in the Xe target. These events are generated through electromagnetic interactions from photons or electrons. The energy window for ER events differs from that of NR events due to differences in scintillation and ionization yield for each type of event. The $3.4-25 \mathrm{keV}_{n r} \mathrm{NR}$ energy range has an $\mathrm{S} 1$ yield range equivalent to $0.9-5.3 \mathrm{keV}_{e e}$, where the "ee" subscript denotes an energy calibration for ER events. The ER energy range 0.9-5.3 $\mathrm{keV}_{e e}$ is therefore taken as the WIMP search background range for ER events.

ER events are created mainly by $\gamma$ rays interacting in the $250 \mathrm{~kg}$ active volume. Gamma rays are generated from the decay of radioisotope impurities in detector construction materials, with typical energies ranging from $\sim 100 \mathrm{keV}$ to several $\mathrm{MeV}$. The dense liquid Xe target $\left(2.9 \mathrm{~g} \mathrm{~cm}^{-3}\right)$ attenuates $\gamma$ rays of these energies at the outer edge of the active region, with a mean free path on the order of several $\mathrm{cm}$. Gamma rays generated outside of the detector are suppressed below significance by the use of a 300 tonne water shield and 20 tonne external steel shield. The total water shielding thickness on all sides is $>2.5$ meters thick.

ER events are also generated by radioisotope decays within the Xe target itself. These isotopes are referred to as "intrinsic." Intrinsic isotopes generate $\beta$ rays or $\mathrm{X}$ rays that are fully absorbed within $\mathrm{mm}$ of the decay site. These isotopes are thoroughly mixed by convection and diffusion, and are distributed homogeneously in the active region. These energies of the $\beta$ rays or X-rays can fall within the $0.9-5.3 \mathrm{keV}_{e e}$ WIMP search energy range.

A subdominant background is expected from NR signatures from neutron scatters. Neutrons are generated internally in the detector through $(\alpha, n)$ interactions in construction materials, and from spontaneous fission predominantly of ${ }^{238} \mathrm{U}$. These neutrons are generated with energies on the scale of $\mathrm{MeV}$, with a mean free path of order $10 \mathrm{~cm}$ in liquid Xe. Neutrons are also generated from muon interactions in the laboratory and water shield. Muon-induced neutrons have energy at the $\mathrm{GeV}$ scale, with a mean free path in liquid Xe much longer than the size of the detector.

LUX uses S1 and S2 signal characteristics for multiple background rejection techniques. Scattering vertex positions in the detector are reconstructed with $\mathrm{cm}$ accuracy in XY, and $\mathrm{mm}$ accuracy in $\mathrm{Z}$. This was measured using XYZ-uniform krypton and tritium calibration data. The accuracy in $\mathrm{Z}$ is tied to the width of an $\mathrm{S} 2$ signal and the drift speed of electrons in LUX of $1.5 \mu \mathrm{m} / \mathrm{s}$ This allows rejection of multiple scatter (MS) events, and enables the use of an inner fiducial region in which to conduct the WIMP search. The fiducial region excludes background events at the detector edges and maximizes WIMP signal-to-noise. Due to the limited $\gamma$ ray mean free path, together with the detector dimensions of $54 \mathrm{~cm}$ in height and $49 \mathrm{~cm}$ in diameter and use of an inner fiducial volume, the number of single-scatter $\gamma$ rays passing through a fiducial volume with radius $18 \mathrm{~cm}$ and height $40 \mathrm{~cm}$ is four orders of magnitude less than the number of $\gamma$ rays with shallow penetration. The ratio of S2 to S1 also provides $99.6 \%$ discrimination against ER events on average over the WIMP search energy range, at 50\% NR acceptance.

This work details modeling and measurements of the LUX background rate from both electromagnetic and neutron sources. Monte Carlo simulation studies of all background components and direct measurement of signatures of these components in data are described in Sec. 2. The characterization of ER background rejection using the S2/S1 ratio is described in Sec. 3. Comparison of expected and measured low-energy background spectra is described in Sec. 4.

\section{Background Modeling}

The LUX background model is comprised of multiple contributions. Each background source has been determined by direct measurements of LUX data, or from sampling of the Xe itself during the run. These measurements are used to normalize Monte Carlo models of each background source. The Monte Carlo models are then used to project the expected low-energy background rate in the data. The details of the background model components, and their constraints from data, are described in this section.

\subsection{LUX Detector Geometry}

A detailed description of the LUX detector can be found in [1]. Not all detector components are equally relevant to the background model however; typically relevance decreases with mass and distance from the active xenon volume. For the purpose of this study, the dominant contributions come from the xenon itself (intrisic sources in the liquid), the PMTs, the PTFE covering the walls of the active volume chamber, the copper for the PMT arrays and radiation shielding, the titanium cryostat, and the thermal insulation inside the vacuum space between the two concentric titanium vessels. Other contributions were also considered, as is discussed later in the text, but those make up the vast majority of the neutron and gamma background in the xenon.

\subsection{LUXSim}

LUX background modeling work was performed using the GEANT4-based LUXSim Monte Carlo simulation package $[6,7]$. The LUXSim package features a reconstruction of the full LUX detector based on CAD designs. The simulation geometry features all components with significant mass or relevance to photon collection modeling. The simulation also incorporates the LUX water shield, 
which thus enables accurate modeling of $\gamma$ ray and neutron scattering and moderation within the main detector. The high-fidelity representation plays an important role in the modeling of low-energy ER and NR background contributions from different detector components, as well as the effects of shielding in determining the $\gamma$ ray and neutron spectra incident at the active region. Simulation results were checked extensively using analytic calculations of signal distributions, based on particle trajectories and mean free paths in different detector components. A rendering of the LUXSim geometry is shown in Fig. 1.

LUXSim also faithfully reproduces detector signals from ER and NR events. LUXSim incorporates the Noble Element Simulation Technique (NEST) package [8], along with several custom physics processes. These processes extend the GEANT4 simulation to generate scintillation photons and ionization electrons in the active region, and are capable of reproducing LUX S1 and S2 waveforms. For all radiogenic backgrounds in this work, LUXSim was used to record only energy depositions, without the creation of scintillation and ionization signals. NEST is used with LUXSim to obtain the final low-energy S1 spectrum for direct comparison with measured data.

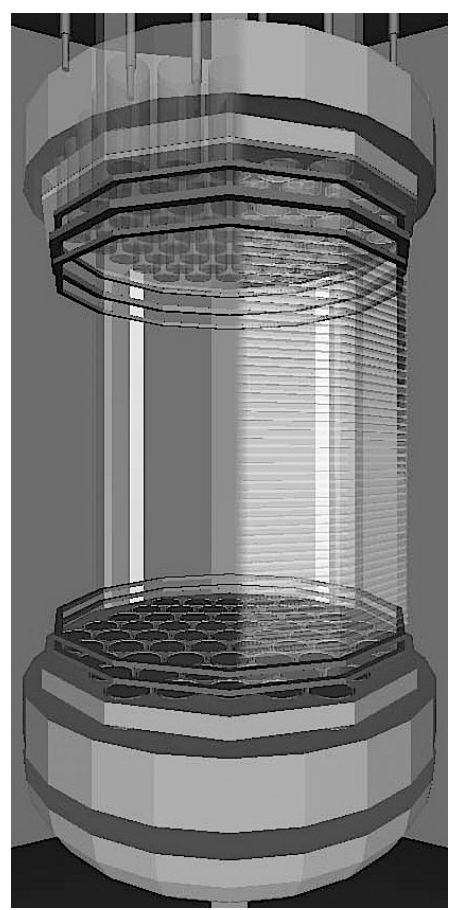

Figure 1: Rendering of the LUX detector from the LUXSim simulation package. LUXSim is used for all studies of LUX radiogenic backgrounds. All geometry components are shown with semitransparency. All high-mass detector construction materials are included in the simulation, as well as the external water shield. Details of all LUX geometry components are given in [1]. Plastic paneling and field shaping rings are cut away from the left side of the image to more clearly show the detailed structure of the PMT arrays. The cryostats are removed for clarity.

\subsection{Gamma Rays from Construction Materials \\ 2.3.1. Material Screening}

LUX construction materials were assayed for their radioactive content before use in detector construction. The materials were screened by high-purity Ge detectors at the Soudan Low-Background Counting Facility (SOLO) [9] and Berkeley Oroville facility. These facilities in total screened $>75$ material samples in the course of the detector construction phase.

Screening results from counting at these facilities are summarized for high-mass ( $\gtrsim \mathrm{kg}$ ) construction materials in Tables 1 and 2. The materials chosen represent $>95 \%$ of the detector dry mass contained within (and including) the cryostats. Photomultiplier tube (PMT) screening results are described in further detail in [9]. Titanium cryostat material screening and cosmogenic activation studies are described in detail in [10] and summarized in Sec. 2.3.2.

Screening results are reported for ${ }^{238} \mathrm{U}$ and ${ }^{232} \mathrm{Th}$ chain isotopes for all materials. For materials that commonly feature ${ }^{40} \mathrm{~K}$ or ${ }^{60} \mathrm{Co}$ contamination, screening measurements or upper limits are also reported for these isotopes. No other radioisotope signatures were discovered during counting, with the exception of cosmogenic ${ }^{46} \mathrm{Sc}$ in Ti. Results are reported for some materials for both ${ }^{238} \mathrm{U}$ and ${ }^{226} \mathrm{Ra}$, corresponding to the first and second halves of the full ${ }^{238} \mathrm{U}$ decay chain. Materials were not subject to further searches for chain disequilibrium due to the relatively low neutron yield from the first half of the ${ }^{238} \mathrm{U}$ chain [11]. Neutron backgrounds are further constrained by direct measurement of neutron MS events in Sec. 2.7. Only the cryostat material shows a significant ${ }^{238} \mathrm{U}$ disequilibrium condition. The predicted neutron background from this is discussed in Sec. 2.7.

The ${ }^{232}$ Th activity was measured using the $511 \mathrm{keV}$ and $583 \mathrm{keV}$ lines from ${ }^{208} \mathrm{Tl}$. As this isotope is the last in the ${ }^{232} \mathrm{Th}$ decay chain, this leaves the potential for ${ }^{232} \mathrm{Th}$ chain disequilibrium in detector materials. Evidence of this disequilibrium condition is seen from high-energy $\gamma$ ray measurements in Sec. 2.3.3. The potential increase in neutron backgrounds from the observed disequilibrium is negligible, and is constrained by the neutron MS event search in Sec. 2.7.

\subsubsection{Cosmogenic Activation of Construction Materials}

The LUX detector was assembled and operated at the Sanford Surface Laboratory over a two year period, before installation in the Davis Underground Laboratory. The operation of the LUX detector at the Sanford Surface Laboratory resulted in the cosmogenic activation of $\mathrm{Ti}$ and $\mathrm{Cu}$ detector construction materials. The activation products of concern with respect to detector backgrounds are ${ }^{46} \mathrm{Sc}$, generated in $\mathrm{Ti}$, and ${ }^{60} \mathrm{Co}$, generated in $\mathrm{Cu}$. Both of these isotopes have non-negligible half-lives ( ${ }^{46} \mathrm{Sc} 84$ days; ${ }^{60}$ Co 5.3 years), and decay modes that can generate WIMP search ER backgrounds. Cosmogenic activation was stopped by moving the detector underground before the beginning of WIMP search running. 


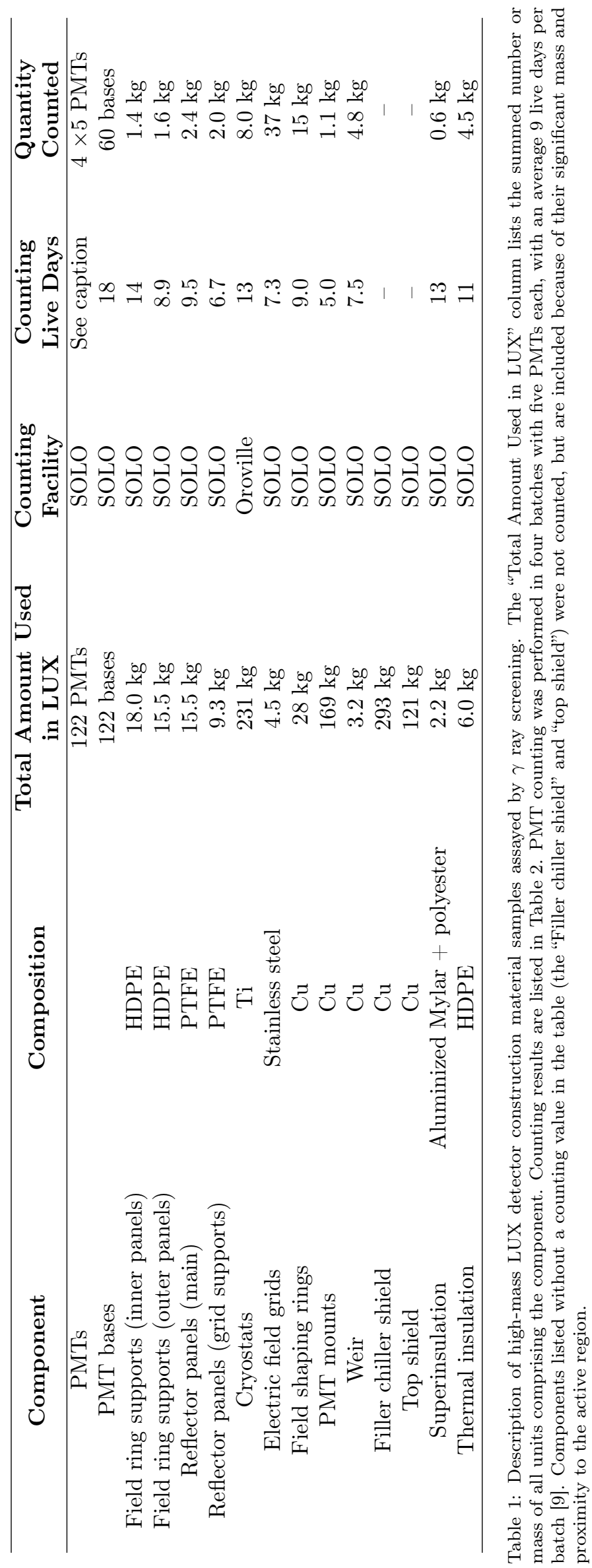



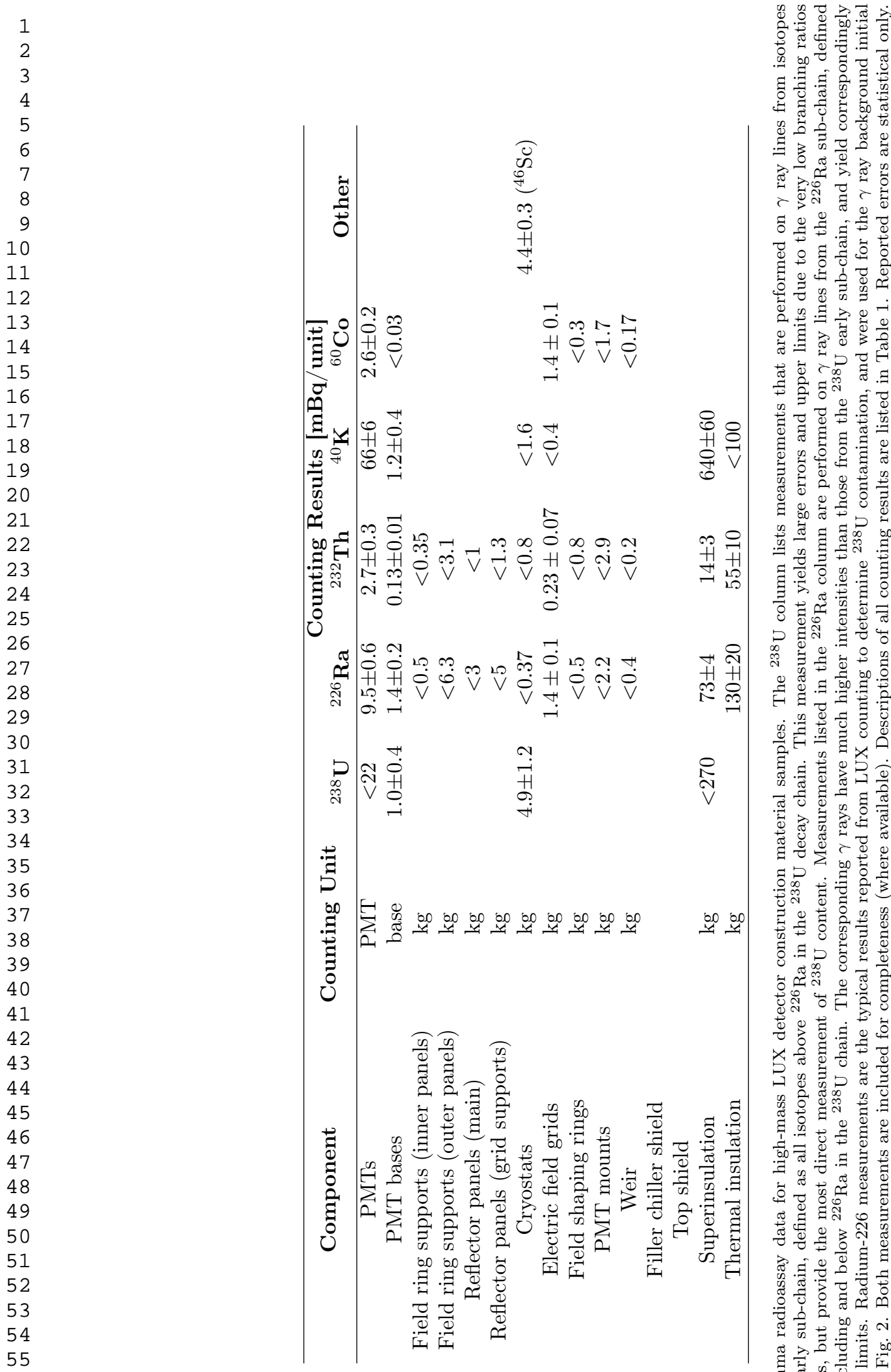

品

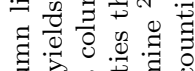

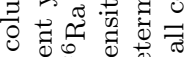
중. 宊

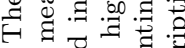

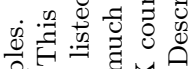

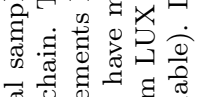

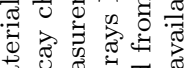

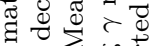

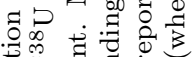

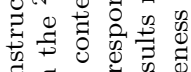

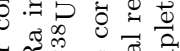

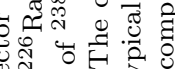
范范 ने द्व

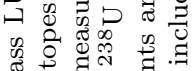
蕰 에

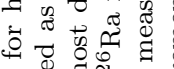
苋节 뜬

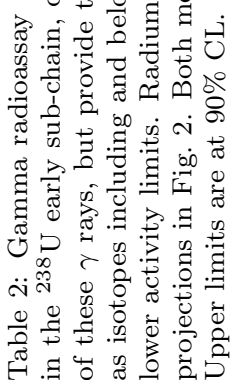


The LUX cryostats were selected from extremely lowradioactivity Ti stock. Counting results and activation studies are reported in [10]. Titanium produces one transient radioisotope, ${ }^{46} \mathrm{Sc}$, from both muon capture and neutron spallation channels. Scandium- 46 produces two simultaneous $\gamma$ rays with energies $889 \mathrm{keV}$ and $1121 \mathrm{keV}$.

A $6.7 \mathrm{~kg}$ control Ti sample was used to estimate the total concentration of ${ }^{46} \mathrm{Sc}$ produced in the LUX cryostats. The sample was screened at the SOLO facility after two years underground, and then transported by ground to the Sanford Surface Laboratory. The sample was activated over a six-month period before being transported by ground back to SOLO for re-analysis. Counting yielded a measurement of $4.4 \pm 0.3 \mathrm{mBq} \mathrm{kg}{ }^{-1}{ }^{46} \mathrm{Sc}$. The measured decay rate was consistent with predictions based on the ACTIVIA simulation package [12], discussed in detail in Sec. 2.4.1.

Based on these measurements, the total ${ }^{46} \mathrm{Sc}$ decay rate in the cryostats immediately after bringing the detector underground on July 12, 2012 was 1.3 Bq, conservatively assuming that the measured ${ }^{46} \mathrm{Sc}$ content after the sixmonth exposure represented $75 \%$ of the equilibrium activation value after the full LUX exposure. The elapsed time between moving LUX underground and beginning the 85.3 day WIMP search run was 284 days, and the 85.3 day WIMP search run was conducted over a period of 109 calendar days. The decay rate averaged over the 85.3 day run was $85 \pm 8 \mathrm{mBq}$. The incident $\gamma$ ray flux from this source at the active region is below the level of measurement due to shielding from other internal materials, preventing a positive measurement of the ${ }^{46} \mathrm{Sc}$ signature in data.

The LUX detector uses $620 \mathrm{~kg}$ of ultra-low-activity oxygen-free high-thermal conductivity $\mathrm{Cu}$ in several construction components. These components include PMT array mounts, $\gamma$ ray radiation shields, thermal insulation, and field shaping rings. A review of $\mathrm{Cu}$ activation studies in [13] is used to estimate the ${ }^{60} \mathrm{Co}$ production levels in LUX. A variation of a factor $\times 4$ is found from all considered studies. From the distribution of results, the expected activation rate at sea level for ${ }^{60} \mathrm{Co}$ in $\mathrm{Cu}$ is taken to be $62 \pm 29 \mathrm{~kg}^{-1} \mathrm{day}^{-1}$. The production rate is assumed to scale by a factor $\times 3.4$ above that at sea level, tracking with the increase in muon-induced neutron flux at Sanford Surface Laboratory surface altitude [14]. The change in neutron spectrum at Sanford Surface Laboratory altitude relative to sea level is assumed to have a subdominant effect on the activation rate. The estimated production rate of ${ }^{60} \mathrm{Co}$ in LUX internals is then $210 \pm 100 \mathrm{~kg}^{-1}$ day ${ }^{-1}$. The estimated total exposure time for the $\mathrm{Cu}$ internals at the Sanford Surface Laboratory is 800 days, leading to a total decay rate of $1.0 \pm 0.5 \mathrm{mBq} \mathrm{kg}^{-1}$ at the time the detector was taken underground. Counting results in Table 2 for $\mathrm{Cu}$ components include upper limits on the presence of ${ }^{60} \mathrm{Co}$ before the components arrived at Sanford.

\subsubsection{High-Energy Measurements and Model Fitting}

LUXSim $\gamma$ ray energy deposition spectra were generated as described in Sec. 2.2. The simulated spectra were compared with measured data to refine the estimate of ${ }^{238} \mathrm{U},{ }^{232} \mathrm{Th},{ }^{40} \mathrm{~K}$, and ${ }^{60} \mathrm{Co}$ concentration in detector internals. Energy spectra in the active region from each of these isotopes were simulated separately in the top (top PMT array), bottom (bottom PMT array), and lateral (PTFE / HDPE paneling) components adjacent to the active region. The isotope decay rate per volume was held constant between PTFE and HDPE components for the latter case.

The decay rate of each isotope in each region was varied independently to obtain the best fit to the measured $\gamma$ ray spectrum as a function of position in the active region. The fit included all energies above $500 \mathrm{keV}_{e e}$, avoiding influence from activated Xe spectra at peak energies of $408 \mathrm{keV}_{e e}$ and below. The measured $\gamma$ ray energy spectrum in the full drift region is compared with LUXSim model estimates in Fig. 2. A two cm volume is removed from the top and bottom to avoid contamination of the dataset from events in irregular field regions. No radial cut is used, because the XY event position reconstruction was found to be skewed for high-energy events due to PMT saturation. Both the initial model based on screening data and the best fit model are shown. The reconstructed energy resolution was fit with the curve $\sigma / \mu=0.59 \cdot E^{-1 / 2}+0.03$.

The measured spectrum in Fig. 2 includes both SS and MS events falling within the active region position cut. Energies for SS events were reconstructed from a combined $\mathrm{S} 1+\mathrm{S} 2$ energy scale, based on calibration values for photon and ionization electron collection efficiency. Energies for MS events were reconstructed in several steps. The initial energy estimate for each vertex was made from the calibrated S2 signal size, and a comparison with lookup tables for ionization electron yield as a function of energy derived from NEST simulations. The total observed S1 signal was then apportioned to each vertex based on the initial estimate of the vertex energy. The event energy was then taken to be the sum of the combined $\mathrm{S} 1+\mathrm{S} 2$ energies from each vertex.

The best-fit simulation peak sizes show good agreement with observed data, with the exception of a $50 \%$ predicted excess of the $969 \mathrm{keV}$ line from ${ }^{228} \mathrm{Ac}\left({ }^{232} \mathrm{Th}\right.$ chain). Agreement with the ${ }^{228}$ Ac peak cannot be found while preserving agreement with peaks at higher and lower energies, unless ${ }^{232} \mathrm{Th}$ chain equilibrium is broken and the ${ }^{228} \mathrm{Ac}$ rate is reduced independently. Equilibrium breakage for ${ }^{228} \mathrm{Ac}$ alone is not a reasonable model, since ${ }^{228} \mathrm{Ac}$ has a 6.2 hour half-life, and would regain its equilibrium concentration on this timescale. The deficit of ${ }^{228} \mathrm{Ac}$ would suggest removal of the parent ${ }^{228} \mathrm{Ra}$ from construction materials, e.g. during manufacturing processes. A detailed model of the isotope concentration was not investigated, since the presence of $50 \%$ excess ${ }^{228} \mathrm{Ac}$ in the model does not affect $\gamma$ ray or neutron background predictions in the 
WIMP search energy range.

The decay rates obtained from the best fit model are given in Table 3 . The total ${ }^{238} \mathrm{U},{ }^{232} \mathrm{Th}$ and ${ }^{40} \mathrm{~K}$ radioisotope content was found to be within one standard deviation of the predicted concentration from material screening, although individual concentrations in the various materials were seen to vary by several standard deviations, and were seen to be partially degenerate in the fit between the different simulated components. ${ }^{60} \mathrm{Co}$ was found to have an excess consistent with a $1.7 \pm 1.0 \mathrm{mBq}$ concentration in $\mathrm{Cu}$ construction materials, in agreement with the predicted rate in Sec. 2.3.2.

The ER background in the WIMP search energy range 0.9-5.3 $\mathrm{keV}_{e e}$ is shown as a function of position from the $\gamma$ ray energy deposition spectra in Fig. 3. The decay rates are normalized to the best-fit results listed in Table 3 . The $\gamma$ ray low-energy continuum is flat, and the background rates in units of $\mathrm{DRU}_{e e}$ are independent of the exact energy window for the WIMP search.

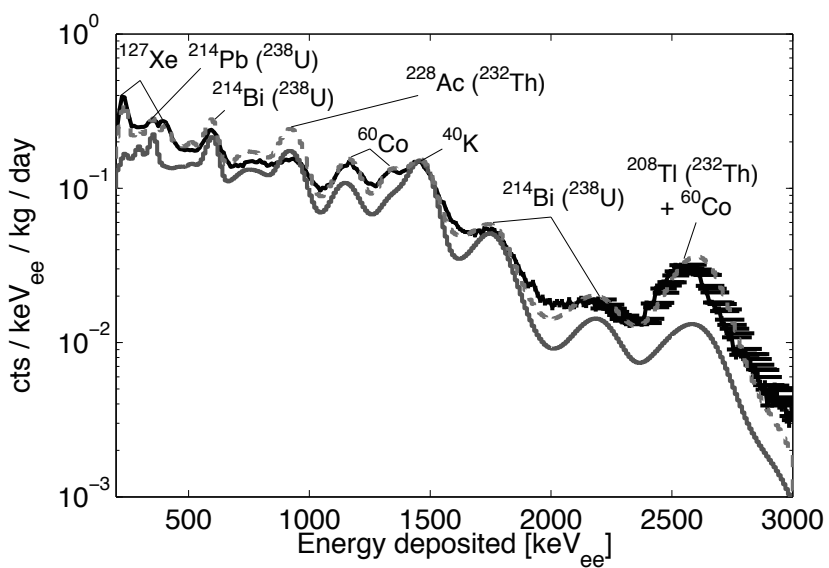

Figure 2: Measured $\gamma$ ray spectrum in the LUX drift region (black), with peak identification labels. A $225 \mathrm{~kg}$ fiducial volume is used for the analysis, removing the top and bottom $2 \mathrm{~cm}$ of the drift region, and using no radial cut. Data includes both SS and MS events. Event energies are reconstructed from the combination of S1 and S2 signals, as described in the text. Horizontal error bars are shown, representing systematic uncertainties in energy reconstruction for high-energy events. Two simulation spectra are shown for comparison. A spectrum based on positive counting measurements alone is shown in gray solid. The spectrum with best-fit scaling for ${ }^{238} \mathrm{U},{ }^{232} \mathrm{Th},{ }^{40} \mathrm{~K}$, and ${ }^{60} \mathrm{Co}$ decays, with independent rates in top, bottom, and side regions of the detector, is shown as gray dashed (red, in color). Fitting was performed for energies above $500 \mathrm{keV}_{e e}$. Energies below $500 \mathrm{keV}_{e e}$ are shown to illustrate the continued agreement between $\gamma$ ray spectra and measured data below the fitting threshold. The spectrum shown has a lower bound at $200 \mathrm{keV}_{e e}$. Best-fit decay rates are listed in Table 3.

\subsection{Cosmogenic Xenon Radioisotopes \\ 2.4.1. Production Models}

The rate of production of noble element radioisotopes in Xe due to cosmic ray exposure was assessed using the ACTIVIA simulation package [12]. The ACTIVIA code modeled isotope production in natural Xe after a 150 day

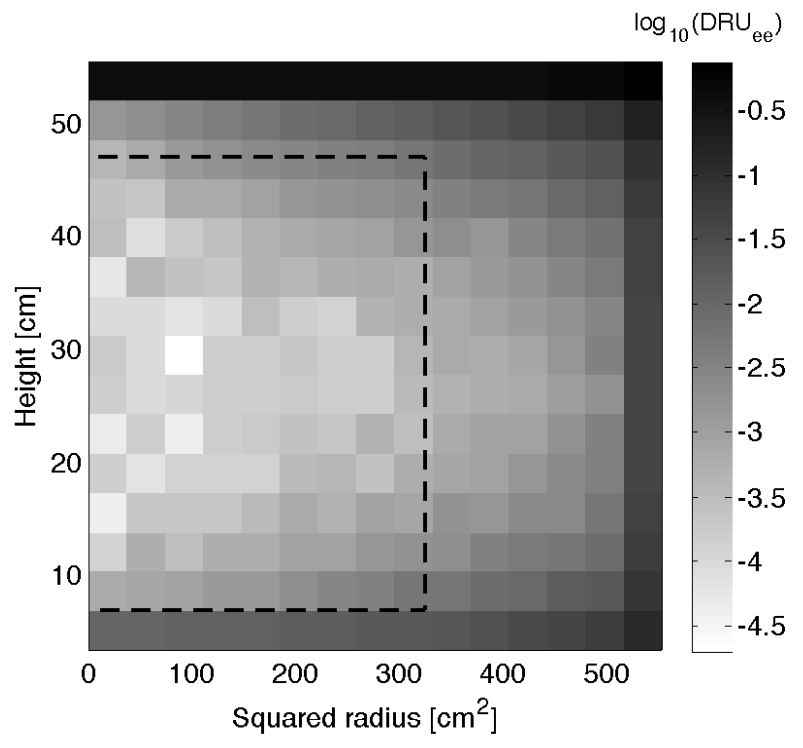

Figure 3: LUX $\gamma$ ray ER background density in the range 0.9$5.3 \mathrm{keV}_{e e}$ as a function of position, extrapolated from high-energy measurements based on Monte Carlo spectra. Rates are in units of $\log _{10}\left(\mathrm{DRU}_{e e}\right)$. The $118 \mathrm{~kg}$ fiducial volume used in the 85.3 day WIMP search run is overlaid as the black dashed contour.

\begin{tabular}{cccc}
\hline Region & Isotope & $\begin{array}{c}\text { Screening } \\
\text { Estimate [Bq] }\end{array}$ & $\begin{array}{c}\text { Best } \\
\text { Fit [Bq] }\end{array}$ \\
\hline \hline \multirow{4}{*}{ Bottom } & ${ }^{238} \mathrm{U}$ & $0.58 \pm 0.04$ & $0.62 \pm 0.16$ \\
& ${ }^{232} \mathrm{Th}$ & $0.16 \pm 0.02$ & $0.23 \pm 0.06$ \\
& ${ }^{40} \mathrm{~K}$ & $4.0 \pm 0.4$ & $2.7 \pm 0.7$ \\
& ${ }^{60} \mathrm{Co}$ & $0.16 \pm 0.01$ & $0.22 \pm 0.06$ \\
\hline \multirow{4}{*}{ Top } & ${ }^{238} \mathrm{U}$ & $0.58 \pm 0.04$ & $0.87 \pm 0.22$ \\
& ${ }^{232} \mathrm{Th}$ & $0.16 \pm 0.02$ & $0.25 \pm 0.06$ \\
& ${ }^{40} \mathrm{~K}$ & $4.0 \pm 0.4$ & $3.8 \pm 1.0$ \\
& ${ }^{23} \mathrm{Co}$ & $0.16 \pm 0.01$ & $0.30 \pm 0.08$ \\
\hline \multirow{3}{*}{ Side } & ${ }^{232} \mathrm{U}$ & $0.94 \pm 0.14$ & $0.22 \pm 0.06$ \\
& ${ }^{20} \mathrm{~T}$ & $0.36 \pm 0.07$ & $1.5 \pm 0.38$ \\
& ${ }^{60} \mathrm{Co}$ & $1.4 \pm 0.1$ & $2.4 \pm 0.6$ \\
& & - & $0.36 \pm 0.09$ \\
\hline
\end{tabular}

Table 3: Screening estimate and best-fit activity values for radioisotopes modeled in high-energy $\gamma$ ray analysis. Screening estimate values are taken from SOLO screening results for the PMTs and grids (corresponding to top and bottom regions), and superinsulation and plastic thermal insulation (side region). Materials with upper limits are not incorporated into the initial estimate. Errors on the best fit values are estimated to be $25 \%$. 
exposure at sea level. Only noble elements were considered, as the LUX purification system is presumed to suppress the concentration of non-noble radioisotopes below significance $[15,16]$.

The short-term exposure history of the LUX Xe is well known. From April 2012 to December 2012, the Xe was located at Case Western Reserve University (altitude $200 \mathrm{~m}$ ) in a basement laboratory, where it was processed for $\mathrm{Kr}$ removal as discussed in Sec. 2.6. The Xe was shipped by ground to Sanford in separate batches and stored above ground (altitude $1.6 \mathrm{~km}$ ), before being brought underground on January 30, 2013. This adds up to roughly half the total Xe load in LUX having spent 49 days at Sanford Aboveground Laboratory altitude, and the other half having spent 7 days at that altitude. Reference [14] provides some guidance for how to scale the cosmic-induced neutron flux and spectrum with altitude, which can be input into activation simulations. However, the effect of immediate surroundings in the lab can introduce an important systematic error in particular on the flux of thermal neutrons incident on the Xe. LUX does not have measurements of the thermal neutron flux at the various relevant locations. In the calculations below, the sea-level activation results from ACTIVIA were used as a starting point. Separate simulation results from 49 days and seven days exposure at Lead, SD altitude for the appropriate Xe masses were added using the neutron flux correction factor of $\times 3.4$ from [14]. The uncertainty on the neutron flux and spectrum was then treated as a systematic. Because thermal neutron capture is the dominant process for the creation of several of the relevant isotopes, this results in a factor $\times 10$ to $\times 100$ uncertainty on the activities. ACTIVIA itself provides a factor $\times 2$ uncertainty due to activation model parameters variations.

The final activity estimates are listed in Table 4, for isotopes with concentrations $>10^{-5}$ after 90 days underground. All the predicted isotopes in the table are identified in initial low-background LUX data, and are discussed in Sec. 2.4.2. A reasonable agreement for all isotopes (within a factor $\times 2$ ) is found when applying an empirical corrective factor of $\times 8$ to all estimates. This may represent an increased thermal neutron flux incident on the $\mathrm{Xe}$ due to the presence of moderating materials in close proximity to the active region, e.g. the $1 \mathrm{~m}$ thick water shield used during the run of the LUX detector at the Sanford Aboveground Laboratory [17]. The numbers reported in Table 4 are the ACTIVIA $\times 8$ values.

\subsubsection{Measurement in LUX Data}

Signatures of ${ }^{129 m} \mathrm{Xe}$ and ${ }^{131 m} \mathrm{Xe}$ isotopes were originally identified in LUX surface run data [17]. After the start of underground operations, the ${ }^{129 m} \mathrm{Xe}$ and ${ }^{131 m} \mathrm{Xe}$ isotope concentration estimates were refined. Two additional activation isotopes, ${ }^{127} \mathrm{Xe}$ and ${ }^{133} \mathrm{Xe}$, were also identified in pre-WIMP run LUX data. The energy spectrum of these isotopes, taken from zero-field data 12 days after the Xe was moved underground and 70 days before

\begin{tabular}{|c|c|c|c|}
\hline Isotope & $\begin{array}{c}\text { Half-life } \\
\text { [Days] }\end{array}$ & $\begin{array}{l}\text { Decay Rate } \\
\text { Predicted }\end{array}$ & $\begin{array}{c}{\left[\mu \mathbf{B q ~ k g}^{-1}\right]} \\
\text { Observed }\end{array}$ \\
\hline${ }^{127} \mathrm{Xe}$ & 36 & 420 & $490 \pm 95$ \\
\hline${ }^{129 m} \mathrm{Xe}$ & 8.9 & 4.1 & $3.2 \pm 0.6$ \\
\hline${ }^{131 m} \mathrm{Xe}$ & 12 & 25 & $22 \pm 5$ \\
\hline${ }^{133} \mathrm{Xe}$ & 5.3 & 0.014 & $0.025 \pm 0.005$ \\
\hline
\end{tabular}

Table 4: Predicted Xe radioisotope activities in units of decay rate per $\mathrm{kg}$ detector target mass, produced from a combination of 150 day exposure at sea level and appropriate exposures at $1.6 \mathrm{~km}$ altitude (details in the text). Predicted and observed decay rates are listed after 90 days underground, where activation has ceased. Isotopes are shown which have a non-negligible concentration after 90 days underground. Activation rates are calculated using ACTIVIA. An overall factor $\times 8$ is applied to all estimates in order to match the measurements, and is thought to represent the error on the thermal neutron flux. Errors on observations are based on uncertainty from peak fitting (Sec. 2.4.2).

the start of the WIMP search run, is shown in Fig. 4. A fitted simulation spectrum is overlaid with contributions from the four Xe cosmogenic isotopes plus an exponential contribution from $\gamma$ ray Compton background. The best-fit decay rates for these spectra correspond to $2.7 \pm 0.5 \mathrm{mBq} \mathrm{kg}{ }^{-1}\left({ }^{127} \mathrm{Xe}\right), 3.6 \pm 0.7 \mathrm{mBq} \mathrm{kg}^{-1}\left({ }^{129 m} \mathrm{Xe}\right)$, $4.4 \pm 0.9 \mathrm{mBq} \mathrm{kg}^{-1}\left({ }^{131 m} \mathrm{Xe}\right)$, and $3.6 \pm 0.7 \mathrm{mBq} \mathrm{kg}^{-1}$ $\left({ }^{133} \mathrm{Xe}\right)$ after 13 days underground.

The decay rates of ${ }^{127} \mathrm{Xe}$ and ${ }^{131 m} \mathrm{Xe}$ were measured by the decay of the $375 \mathrm{keV}_{e e}$ and $164 \mathrm{keV}_{e e}$ peaks, respectively, over the course of the 118 day WIMP search run. The measured peak rates and best-fit models are shown in Fig. 5. The decay fits yield concentrations of $115 \pm 20 \mu \mathrm{Bq} \mathrm{kg}^{-1}{ }^{127} \mathrm{Xe}$ and $7.3 \pm 1.5 \mu \mathrm{Bq} \mathrm{kg}^{-1} 131 m \mathrm{Xe}$, averaged over the second half of the 85.3 day WIMP search run. The concentrations calculated from the peak decays agree with initial projections from early zero-field data estimates.

The measured decay rates of all identified Xe radioisotopes are listed in Table 4 . The measured decay rates are within a factor $\times 2$ of predictions, if one also applies a corrective factor $\times 8$ to all predicted values. This is thought to be related to the uncertainty on the thermal neutron flux, as well as uncertainties inherent to the simulation model. Further studies and measurement are planned in order to try and control these uncertainties.

Useful energy calibration points were found from ${ }^{127} \mathrm{Xe}$, ${ }^{131 m} \mathrm{Xe}$ and ${ }^{129 m} \mathrm{Xe}$. The 5.2 day half-life of ${ }^{133} \mathrm{Xe}$ rendered this isotope unmeasurable by the start of WIMP search running. The only activated Xe isotope capable of generating a significant WIMP search background is ${ }^{127} \mathrm{Xe}$.

\subsection{3. ${ }^{127}$ Xe Backgrounds}

Decays of ${ }^{127}$ Xe generate a low-energy ER background in the LUX 85.3 day WIMP search data. Xenon-127 decays via electron capture, resulting in an orbital vacancy. The vacancy is filled by electron transitions from higher orbitals, resulting in an X-ray or Auger electron cascade. In the case of ${ }^{127} \mathrm{Xe}$, the capture electron comes from the 


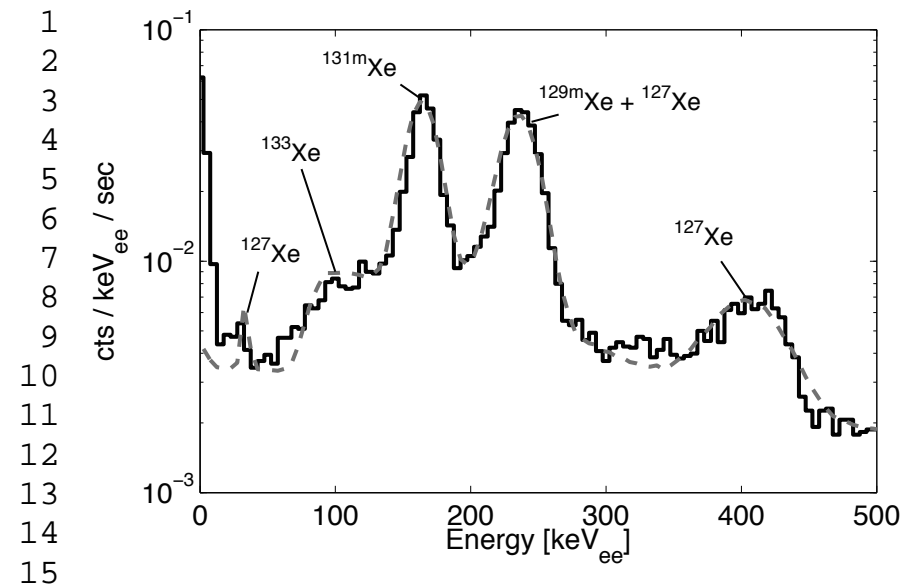

16 Figure 4: Early zero-field LUX data taken 12 days after bringing 17 the Xe underground, featuring peaks from cosmogenically activated

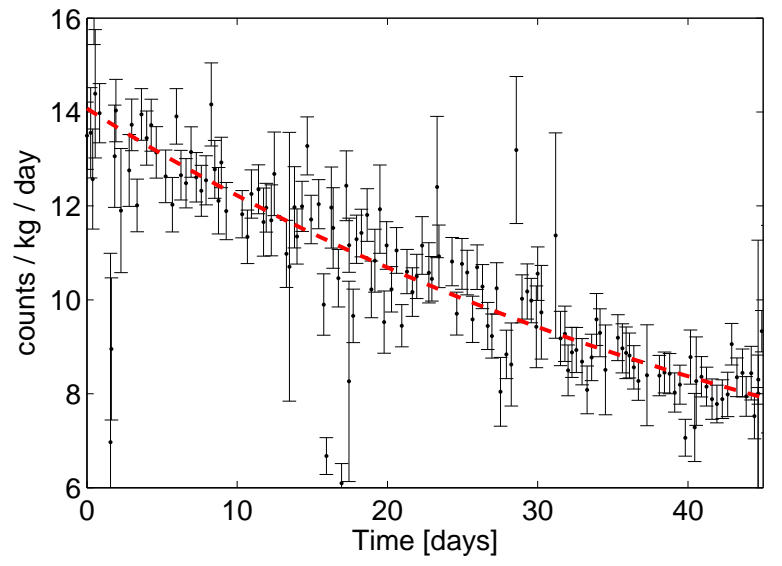

(a)

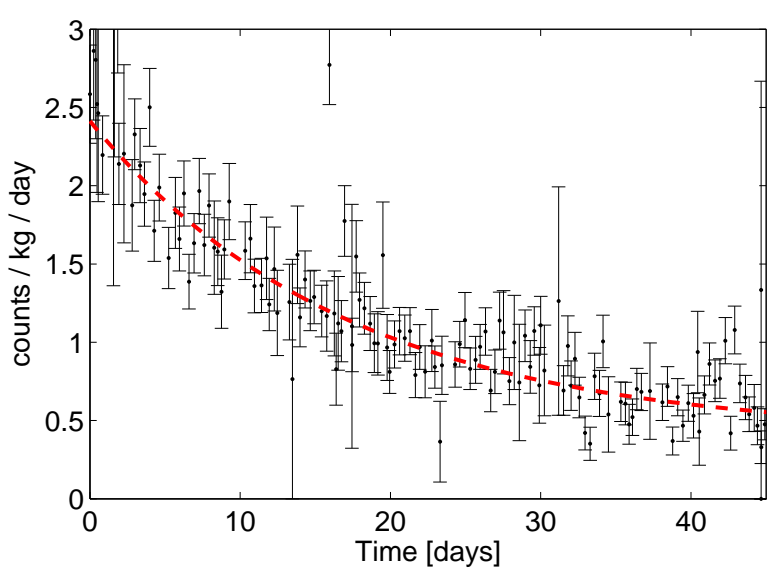

(b)

Figure 5: Activated Xe peak rates as a function of time, measured over part of the 85.3 day WIMP search run, beginning May 1, 2013. Peaks are measured for (a) ${ }^{127} \mathrm{Xe}$, at 375 and $410 \mathrm{keV}_{e e}$, and (b) ${ }^{131 m} \mathrm{Xe}$, at $164 \mathrm{keV}_{e e}$. Best-fit exponential functions are shown with dashed lines (red, in color). The exponential slope of each function is fixed to the corresponding literature half-life listed in Table 4, and is not varied with the fit. Error bars are statistical. 


\subsection{Radon}

\subsubsection{Identification of Radon Daughters in LUX Data}

The decay of ${ }^{222} \mathrm{Rn}$ and ${ }^{220} \mathrm{Rn}$ daughters generates a low-energy ER background in LUX data. Radon isotopes decay through several short-lived daughter stages. ${ }^{222} \mathrm{Rn}$ generates ${ }^{214} \mathrm{~Pb}$ and ${ }^{214} \mathrm{Bi}$. ${ }^{220} \mathrm{Rn}$ generates ${ }^{212} \mathrm{~Pb}$, which decays with a scheme similar to ${ }^{214} \mathrm{~Pb}$. These isotopes undergo "naked" or "semi-naked" $\beta$ decay. Naked $\beta$ decay refers to $\beta$ ray emission without accompanying EM emission that could veto the event. Semi-naked $\beta$ decay refers to $\beta$ ray emission accompanied by emission of a highenergy $\gamma$ ray, which can potentially escape the active region. If the $\gamma$ ray escapes the active region, the $\beta$ ray is not tagged as a background event.

Radon daughters were identified in LUX data through $\alpha$ decay signatures. Alpha particles are clearly distinguished in LUX data by their large S1 signal sizes, ranging from $4 \times 10^{4}-9 \times 10^{4}$ photoelectrons (phe). The S1 pulse sizes from $\alpha$ particle events are much larger than $\mathrm{S} 1$ pulses from $\gamma$ ray events, which reach up to $1.5 \times 10^{4}$ phe. Alpha particles produce a clear signature in LUX data, which can be used to characterize the ${ }^{222} \mathrm{Rn}$ and ${ }^{220} \mathrm{Rn}$ chain decay rates and distributions in the active region. These isotopes are the only sources of $\alpha$ decays in LUX.

Identified $\alpha$ particle peaks are shown in Fig. 6. The six $\alpha$ particle peaks are fit to a sum of five Gaussians and one Crystal Ball distribution [19], which is a Gaussian with a power law tail and is characteristic of lossy processes. The Crystal Ball distribution is used for the ${ }^{210} \mathrm{Po}$ peak, to characterize partial $\alpha$ particle energy loss from transit through materials in contact with the active region. The peak means are scaled for a best fit to literature values of $\alpha$ particle energies from ${ }^{222} \mathrm{Rn}$ and ${ }^{220} \mathrm{Rn}$ daughters. The total measured daughter rates were taken from the best-fit peak areas. The radon daughter isotopes, $\alpha$ particle energies, half-lives, and measured decay rates are summarized in Table 5.

The reconstruction efficiency for short-lived isotopes is limited due to overlap with the parent event. The efficiency for ${ }^{214} \mathrm{Po}$ event reconstruction was estimated at $52 \%$, based on the efficiency of $\mathrm{S} 2$ pulse separation between the ${ }^{214} \mathrm{Po}$ and the parent ${ }^{214} \mathrm{Bi} \alpha$ particle events. Poor reconstruction efficiency was found for overlapping ${ }^{212} \mathrm{Bi} /{ }^{212} \mathrm{Po}$ events, which comprise virtually all ${ }^{212} \mathrm{Po}$ decay events due to its $0.3 \mu$ s half-life. No ${ }^{212}$ Po measurement is reported for this reason.

The reconstructed energies of all $\alpha$ particle decays are taken to be the total Q-values of the decays, accounting for both the $\alpha$ particle and the recoiling daughter nucleus. The exception is ${ }^{210} \mathrm{Po}$, which decays on detector surfaces; this is because the parent ${ }^{210} \mathrm{~Pb}$ is sourced dominantly from the PTFE surfaces, and has negligible concentration in the active region due to removal by the purification system. For ${ }^{210} \mathrm{Po}$, it is assumed that the reconstructed energy is the $\alpha$ particle energy only. If the ${ }^{210} \mathrm{Po} \alpha$ particle is detected in the active region, then the recoiling
${ }^{206} \mathrm{~Pb}$ nucleus becomes further embedded in the material surface and does not produce a visible signal. If the ${ }^{206} \mathrm{~Pb}$ nucleus recoils into the active region, then the $\alpha$ particle will be emitted into the PTFE and will not contribute to the observed 5.3 MeV peak.

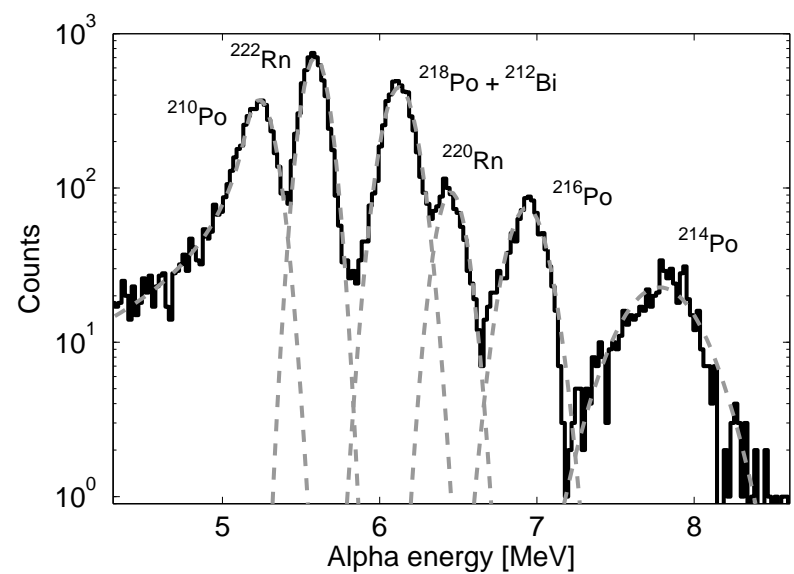

Figure 6: Signatures of $\alpha$ particle decays in LUX WIMP search data in the active region. Energy is reconstructed from S1 measurements, calibrated on the observed location of the ${ }^{222} \mathrm{Rn}$ peak. Counts are collected over 6 livedays, spaced periodically over the course of the 85.3 day WIMP search run. Measured data (black) are fitted with Gaussian curves, with the exception of the ${ }^{210}$ Po peak which is fit with a Crystal Ball distribution (see text for discussion). Fitted curves are shown in gray dashed (peaks shown in color online). Fitted curve peak values are fixed at the Q-values of the $\alpha$ decays for all isotopes, with the exception of ${ }^{210} \mathrm{Po}$ where the peak value is the mean $\alpha$ particle energy.

\subsubsection{Radon Daughter Backgrounds}

Radon daughters that generate low-energy ER backgrounds are not directly countable in LUX data. However, their decay rates can be bounded based on measurements of parent and daughter $\alpha$ decays. The decay rates of ${ }^{214} \mathrm{Bi}$ and ${ }^{214} \mathrm{~Pb}$ are bounded by the measured ${ }^{218} \mathrm{Po}$ and ${ }^{214} \mathrm{Po}$ rates, yielding a range of $3.5-14 \mathrm{mBq}$ in the LUX active region. The ${ }^{212} \mathrm{~Pb}$ rate has an upper bound of $<2.8 \mathrm{mBq}$ from ${ }^{216} \mathrm{Po}$, and is assumed to be further removed from the active region due to its 11 hour half-life. ${ }^{214} \mathrm{Bi}$ is also removed from consideration as an ER background source due to the $160 \mu$ s half-life of the daughter ${ }^{214}$ Po, which creates a $90 \%$ probability of overlap with the ${ }^{214} \mathrm{Po} \alpha$ decay within the LUX $1 \mathrm{~ms}$ event window. The remaining $10 \%$ of events are accounted for in the background model, but do not have a significant contribution. The primary isotope of concern with respect to ER backgrounds is ${ }^{214} \mathrm{~Pb}$.

An additional constraint is placed on the ${ }^{214} \mathrm{~Pb}$ rate from direct measurement of the ER spectrum. The measurement is performed in a low-background $30 \mathrm{~kg}$ fiducial volume, where the external $\gamma$ ray depositions are heavily suppressed. The measurement is performed in the range 300-350 $\mathrm{keV}_{e e}$, between the peaks from ${ }^{127} \mathrm{Xe}$. The measured spectrum, along with models of ${ }^{214} \mathrm{~Pb}$ and ${ }^{127} \mathrm{Xe}$ spectra, are shown in Fig. 7. For the purposes of setting 


\begin{tabular}{cccccc}
\hline $\begin{array}{c}\text { Decay } \\
\text { Chain }\end{array}$ & Isotope & $\begin{array}{c}\text { Energy } \\
{[\mathrm{MeV}]}\end{array}$ & $\begin{array}{c}\text { Measured } \\
\text { Energy [MeV] }\end{array}$ & Half-life & Event Rate [mHz] \\
\hline \hline \multirow{2}{*}{${ }^{238} \mathrm{U}$} & ${ }^{222} \mathrm{Rn}$ & 5.59 & $5.59 \pm 0.08$ & $3.8 \mathrm{~d}$ & $17.9 \pm 0.2$ \\
& ${ }^{218} \mathrm{Po}$ & 6.16 & $6.12 \pm 0.10$ & $3.1 \mathrm{~min}$ & $14.4 \pm 0.2^{*}$ \\
& ${ }^{214} \mathrm{Po}$ & 7.84 & $7.80 \pm 0.2$ & $160 \mu \mathrm{s}$ & $3.5 \pm 0.1$ \\
& ${ }^{210} \mathrm{Po}$ & 5.30 & $5.22 \pm 0.09$ & $140 \mathrm{~d}$ & $14.3 \pm 0.2$ (on walls) \\
& & & & & $7.2 \pm 0.2$ (on cathode) \\
\hline \multirow{2}{*}{${ }^{232} \mathrm{Th}$} & ${ }^{220} \mathrm{Rn}$ & 6.41 & $6.47 \pm 0.09$ & $56 \mathrm{~s}$ & $2.6 \pm 0.1$ \\
& ${ }^{216} \mathrm{Po}$ & 6.91 & $6.95 \pm 0.1$ & $0.15 \mathrm{~s}$ & $2.8 \pm 0.1$ \\
& ${ }^{212} \mathrm{Bi}$ & 6.21 & $6.12 \pm 0.10$ & $61 \mathrm{~min}$ & $14.4 \pm 0.2^{*}$ \\
& ${ }^{212} \mathrm{Po}$ & 8.83 & - & $0.30 \mu \mathrm{s}$ & - \\
\hline
\end{tabular}

${ }^{*}$ Rates due to the sum of ${ }^{218} \mathrm{Po}$ and ${ }^{212} \mathrm{Bi}$ activities.

Table 5: Radon chain daughter isotopes measured in the LUX active volume during the 85.3 day WIMP search run. Measurements are collected over 6 livedays, spaced periodically over the course of the 85.3 day WIMP search run. The known energies are the decay Q-values, except in the case of ${ }^{210} \mathrm{Po}$, where the listed known energy is the $\alpha$ particle emission energy. ${ }^{218} \mathrm{Po}$ and ${ }^{212} \mathrm{Bi}$ are too close in energy to be resolved as separate peaks. The summed activity values are listed in the table, and no attempt is made to apportion the activities since neither isotope contributes to LUX backgrounds. Errors are statistical, and are reported at $\pm 34 \%$.

a limit on the ${ }^{214} \mathrm{~Pb}$ decay rate, it is conservatively assumed that there is no $\gamma$ ray background in this range and that all activity is due to ${ }^{214} \mathrm{~Pb}$ decay. The ${ }^{214} \mathrm{~Pb}$ decay spectrum is compared with data in this range, using the nearby ${ }^{127} \mathrm{Xe}$ peaks to calibrate cut efficiencies and estimate energy resolution. The upper limit on ${ }^{214} \mathrm{~Pb}$ activity from this exercise is $<32 \mu \mathrm{Bq} \mathrm{kg}^{-1}$ at $90 \% \mathrm{CL}$, or $<8 \mathrm{mBq}$ integrated over the entire active region. This exercise provides a much stronger upper limit on the ${ }^{214} \mathrm{~Pb}$ activity than interpolation from $\alpha$ decay rates alone.

The low-energy ER background contribution for ${ }^{214} \mathrm{~Pb}$ is taken from the fraction of its $\beta$ spectrum that falls inside the WIMP search range. This accounts for the ER background generated from naked $\beta$ decay. ${ }^{214} \mathrm{~Pb}$ can also potentially generate a position-dependent background from a semi-naked $\beta$ decay, where the $352 \mathrm{keV} \gamma$ ray escapes from the active region. For the $118 \mathrm{~kg}$ fiducial, the naked $\beta$ ray background component comprises $>95 \%$ of the total background signal. The semi-naked component negligible and is ignored. The total contribution, with a lower bound from the measured ${ }^{214} \mathrm{Po}$ decay rate and an upper bound from the high-energy ER spectrum, is $0.10-0.22 \mathrm{mDRU}_{e e}$. Background models assume a concentration of $0.2 \mathrm{mDRU}_{e e}{ }^{214} \mathrm{~Pb}$, calculated assuming that the ${ }^{222} \mathrm{Rn}$ chain rates progress as a geometric series, with endpoints constrained by the measured decay rates of the visible ${ }^{222} \mathrm{Rn}$ daughters.

\section{6. ${ }^{85} \mathrm{Kr}$ Removal, Monitoring and Backgrounds}

The research-grade Xe procured for LUX contained an average $130 \mathrm{ppb} \mathrm{g} / \mathrm{g}{ }^{\text {nat }} \mathrm{Kr} / \mathrm{Xe}$ upon acquisition. Natural $\mathrm{Kr}$ contains the unstable isotope ${ }^{85} \mathrm{Kr}$ in estimated concentrations of $2 \times 10^{-11}(\mathrm{~g} / \mathrm{g})$ [20]. ${ }^{85} \mathrm{Kr}$ decays with a half-life of 10.8 years via emission of a $\beta$ with $687 \mathrm{keV}$ endpoint. The $\beta$ emission creates low-energy ER backgrounds at the level of $5 \mathrm{DRU}_{e e}$, at $130 \mathrm{ppb}{ }^{\text {nat }} \mathrm{Kr} / \mathrm{Xe}$.

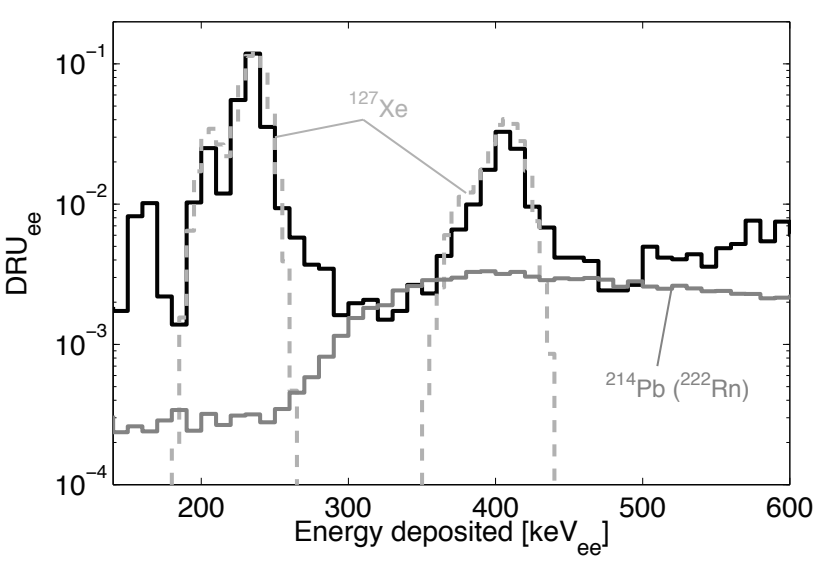

Figure 7: Constraint on ${ }^{214} \mathrm{~Pb}$ in a $30 \mathrm{~kg}$ fiducial volume. Spectra are shown for measured data (black), simulation of ${ }^{127}$ Xe peaks (gray dashed, green in color), and simulation of ${ }^{214} \mathrm{~Pb}$ (gray solid, red in color). The ${ }^{214} \mathrm{~Pb}$ activity shown is $32 \mu \mathrm{Bq} \mathrm{kg}^{-1}$, measured to be the $90 \%$ upper limit from data in the range $300-350 \mathrm{keV}_{e e}$. 
Krypton is not removed by the LUX getter, as the getter removes only non-noble impurities. An independent Kr removal system for LUX Xe was built and operated at Case Western Reserve University. The Kr removal system was established with a goal of reducing ${ }^{85} \mathrm{Kr}$ background levels to $<0.2 \mathrm{mDRU}_{e e}$. This level was chosen so that the ${ }^{85} \mathrm{Kr}$ background would be $\times 0.25$ that of the external $\gamma$ ray background projected for a $100 \mathrm{~kg}$ fiducial volume. The corresponding nat $\mathrm{Kr}$ concentration in $\mathrm{Xe}$ is $<5 \mathrm{ppt}$.

The Kr removal system uses a column of activated charcoal to chromatographically separate Kr from Xe [21]. Details of the system will be released in a separate upcoming LUX publication [22]. From August 2012 to January 2013, a total of $400 \mathrm{~kg}$ of Xe was purified with the charcoal system for the first LUX WIMP search run. Krypton was reduced by an average factor $3 \times 10^{4}$ for each Xe batch. No other impurities were introduced in significant quantities during the purification process. Loss of Xe during the process was negligible. The final measured $\mathrm{Kr}$ concentration in LUX Xe immediately after purification was $4 \pm 1 \mathrm{ppt} \mathrm{g} / \mathrm{g}$.

The purified Xe was sampled weekly over the course of the WIMP search run to detect any new $\mathrm{Kr}$ signatures. Krypton detection at the ppt level was accomplished using a liquid nitrogen cold trap and mass spectrometry analysis, based on the design presented in [23]. Average Kr levels in LUX Xe during the WIMP search run were measured to be $3.5 \pm 1.0 \mathrm{ppt} \mathrm{g} / \mathrm{g}$. The corresponding ER background rate, calculated directly from the ${ }^{85} \mathrm{Kr}$ beta spectrum, is $0.17 \pm$ $0.10 \mathrm{mDRU}_{e e}$. The error on the background estimate is due to uncertainty in the total Kr concentration in the Xe, as well as uncertainty in the ratio of ${ }^{85} \mathrm{Kr} /{ }^{\text {nat }} \mathrm{Kr}$, which is taken to be a factor $\times 2$.

\subsection{Neutrons from Construction Materials}

\subsubsection{Predicted Yields and LUXSim Modeling}

The dominant source of neutrons in LUX is the ${ }^{238} \mathrm{U}$ and ${ }^{232}$ Th content in the R8778 PMTs $[1,9]$. The ${ }^{238} \mathrm{U}$ and ${ }^{232} \mathrm{Th}$ content of the remaining major internal components add up to less than half that of the PMTs, and their contribution to the total neutron rate is subdominant. The $(\alpha, n)$ neutron spectrum generated from the PMTs was calculated using the Neutron Yield Tool developed by LUX collaborators at the University of South Dakota $[24,25]$. The tool was used to predict the total neutron yield based on the exact chemical makeup of the PMT components, obtained via confidential communication with Hamamatsu Photonics K.K. The total neutron generation rate from $(\alpha, n)$ processes and spontaneous fission in the PMTs is $1.2 \mathrm{n} \mathrm{PMT}^{-1} \mathrm{yr}^{-1}$.

An additional contribution to the $(\alpha, n)$ neutron background results from ${ }^{210} \mathrm{~Pb}$ that has plated onto detector materials yields. The dominant component of this additional contribution comes from $\alpha$ particle interactions in PTFE, as fluorine generates $1-3$ orders of magnitude more neutrons through $(\alpha, n)$ reactions than other typical con- struction materials [11]. The observed ${ }^{210} \mathrm{Po} 5.3 \mathrm{MeV} \alpha$ decay rate on the PTFE walls of the active region is $14 \mathrm{mHz}$. The corresponding neutron emission rate, multiplied by $\times 2$ to account for PTFE surfaces not visible to the active region, is $8.8 \mathrm{yr}^{-1}$. This emission rate is only $6 \%$ of the total PMT neutron emission rate. Polonium-210 neutrons are not incorporated into the NR background model.

LUXSim was used to simulate the emission of neutrons isotropically from the top and bottom PMT arrays, using the energy spectrum calculated with the Neutron Yield Tool. MS and SS cuts were implemented in postprocessing of LUXSim data, mimicking the cuts used in WIMP search data. Neutron events were selected in the range $3.4-25 \mathrm{keV}_{n r}$. The total number of $\mathrm{SS}$ neutron events predicted in the WIMP search energy range in 85.3 days $\times 118 \mathrm{~kg}$ fiducial volume is 0.06 .

\subsubsection{Multiple Scatter Identification in LUX Data}

Radiogenic neutron SS events mimic a WIMP signature in both NR energy spectrum and ER/NR discrimination, as described in Sec. 1. For this reason, and because the neutron SS rate is expected to be very low in LUX, a direct search for neutron SS events cannot be used to place meaningful constraints on the neutron background. The $47 \mathrm{~cm}$ LUX diameter creates an environment in which the number of neutron MS events are much greater than the number of neutron SS events, due to the $\lesssim 10 \mathrm{~cm}$ mean free path of typical radiogenic neutrons. Constraints on neutron MS event rates were used to place an upper limit on the neutron SS event rates.

Simulated neutron background studies were used to calculate the ratio of the number of NR MS events within an enlarged $180 \mathrm{~kg}$ fiducial volume to the number of SS events within a smaller WIMP search fiducial volume. For MS events, it was required that the energy-weighted average of all scatter vertices fall within the $180 \mathrm{~kg}$ volume. The $180 \mathrm{~kg}$ fiducial is chosen as the maximum volume where low-energy MS events are reliably reconstructed. WIMP search fiducial volumes with masses $118 \mathrm{~kg}$ and $100 \mathrm{~kg}$ are explored, where the $118 \mathrm{~kg}$ volume corresponds to the fiducial volume used for the 85.3 day WIMP search run, and the $100 \mathrm{~kg}$ volume is a nominal LUX one-year WIMP search run fiducial chosen for consistency with previous background studies. LUXSim simulations estimate a ratio of $13 \mathrm{MS}$ events in the $180 \mathrm{~kg}$ fiducial per one SS event in the $118 \mathrm{~kg}$ fiducial volume.

The MS NR and ER S2/S1 discrimination bands were constructed using NEST and measured LUX efficiencies for S1 and S2 detection [2]. The bands were used to characterize the observed MS events as ER-like or NRlike. The S1 and S2 signals for MS events were defined as the summed, position-corrected S1 and S2 signals from all scattering vertices, as described in Sec. 2.3.3. Construction of the ER MS band used the assumption of a flat-energy, two-scatter population, corresponding to the expected MS background from $\gamma$ ray sources. Construction of the NR MS band used the MS energy depositions 
from LUXSim neutron background studies. Models of the underlying energy spectra are necessary for reconstruction of the expected bands due to the changing average S1 and S2 yields as a function of energy for both ER and NR events.

MS selection cuts mirror the cuts used to select singlescatter NR events in WIMP search data, but requiring events with $>1$ S2 pulse, each with $>200$ phe, and within a S1 range of $0-120$ phe. The cuts are estimated to have a $95 \%$ efficiency in catching all NR MS from the predicted neutron energy spectrum. Candidate MS events are shown along with the $\pm 1.28 \sigma \mathrm{ER}$ and NR bands in Fig. 8 . The NR MS search region was defined below the NR band centroid, giving an overall search efficiency of $48 \%$. No NRlike MS events were observed in the $180 \mathrm{~kg}$ search region in 85.3 days, corresponding to an upper-limit of $2.3 \mathrm{MS}$ events at $90 \%$ CL. A population of events well above both the ER and NR bands are found, which are comprised of events in the gas Xe region, and other events with waveform topologies generally inconsistent with MS events in the $180 \mathrm{~kg}$ search region.

Given the ratio of $13 \mathrm{MS}$ to SS events, and the search efficiency of $48 \%$, an upper-limit of $2.3 \mathrm{NR}$ MS events sets a NR SS event upper limit for the run of $<0.37$ events in the $118 \mathrm{~kg}$ fiducial volume. The expectation based on Monte Carlo results is 0.06 events. For a one-year run using a $100 \mathrm{~kg}$ fiducial, the upper limit is $<0.72$ events, with an expectation of 0.28 .

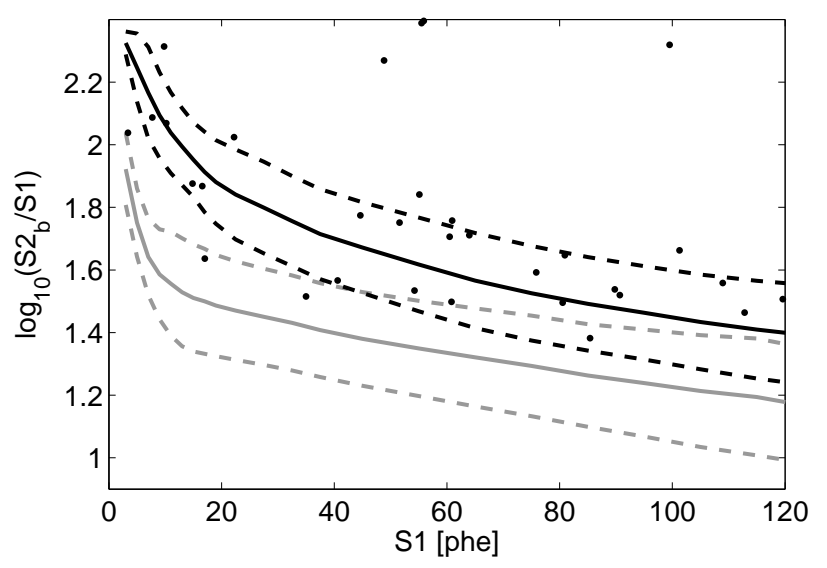

Figure 8: Low-energy MS events in LUX during the 85.3 day WIMP search run, within a $180 \mathrm{~kg}$ fiducial volume. Events are plotted in $\mathrm{ER} / \mathrm{NR}$ discrimination space. The subscript "b" in $\mathrm{S} 2_{b}$ denotes S2 signals from the bottom PMT array only, chosen to avoid irregularities in S2 signals due to deactivated PMTs in the top array. The MS events are used to place an upper limit on the NR SS rate for the 85.3 day and one-year WIMP search runs. Overlaid are the projected bands for ER (black, blue in color) and NR (gray, red in color) MS events, shown with centroids (solid) and $\pm 1.28 \sigma$ bounds (dashed). Events well above the ER band are found to be inconsistent with MS events in the search volume.

\subsection{External Backgrounds}

\subsubsection{Water Shield Design and Radiogenic Cavern Back- grounds}

The LUX detector is placed inside a 300 tonne water shield designed to render all external backgrounds subdominant to internal backgrounds. The design of the water shield was assisted by Monte Carlo simulations of both $\gamma$ ray and neutron external backgrounds. The primary factor driving the size and configuration of the water shield was the reduction of the high-energy muon-induced neutron background to the level of 0.1 WIMP-like events in $100 \mathrm{~kg} \times$ one-year [1]. The cylindrical water shield is $7.6 \mathrm{~m}$ in diameter and $6.1 \mathrm{~m}$ in height, providing a minimum water thickness of $2.75 \mathrm{~m}$ at the top, $3.5 \mathrm{~m}$ on the sides, and $1.2 \mathrm{~m}$ on the bottom. It is built on top of 20 tonnes of lowradioactivity steel plates arranged in an inverse pyramid configuration, with a maximum thickness of $31 \mathrm{~cm}$. The shape of the inverse steel pyramid is optimized to reduce the $\gamma$ ray flux originating from the rock below, reducing the total external $\gamma$ ray rate in the detector by a factor of 40 based on GEANT4 Monte Carlo simulations [26].

The external $\gamma$ ray background is dominated by decays of ${ }^{40} \mathrm{~K}$ and the ${ }^{238} \mathrm{U}$ and ${ }^{232} \mathrm{Th}$ chains in the cavern rock. Radiometric surveys of the Homestake mine indicate that most rock in the $4850 \mathrm{ft}$ level are of the type labeled HST06 , consisting of $0.160 \mathrm{ppm}{ }^{238} \mathrm{U}, 0.200 \mathrm{ppm}{ }^{232} \mathrm{Th}$ and $1540 \mathrm{ppm}{ }^{40} \mathrm{~K}$ [27]. Geological surveys also show rhyolite intrusions in the rock with much higher radioactivities, with average contamination levels of $8.6 \mathrm{ppm}{ }^{238} \mathrm{U}, 10.8$ ppm ${ }^{232} \mathrm{Th}$, and $29000 \mathrm{ppm}{ }^{40} \mathrm{~K}$. The percentage of rhyolite intrusions on the cavern surface is unknown, and in order to set a conservative estimate of the $\gamma$ ray event rate in the detector, background estimates assume a cavern completely composed of rhyolite, resulting in a flux of $9 \gamma \mathrm{cm}^{-2} \mathrm{~s}^{-1}$ at the water shield outer surface. Radioactive screening of typical concrete mixes indicate that the radioactive contamination levels in concrete are in the range of 1-2 ppm and are well below the conservative assumption for the surrounding rock $[28,29]$.

Monte Carlo simulations of the water shield use a "standard rock" $\gamma$ ray energy spectrum obtained from measurements at the Boulby Mine [30] and scaled to match the radioactivity levels assumed for the Davis cavern. The simulations show that $\gamma$ ray flux is reduced by a factor of $2 \times 10^{-10}$ by the water shield and steel pyramid. The resulting $\gamma$ ray flux incident on the LUX detector generates a low-energy $\mathrm{ER}$ event rate of $27 \mathrm{nDRU}_{e e}$ in the active region.

The external neutron flux is dominated by the environmental fast neutron background $(\mathrm{E}>1 \mathrm{MeV})$ due to radioactive processes in the surrounding rock and concrete. The neutrons are mainly produced by ${ }^{238} \mathrm{U}$ spontaneous fission and $(\alpha, \mathrm{n})$ neutrons generated in the rock and concrete from the ${ }^{238} \mathrm{U}$ and ${ }^{232} \mathrm{Th}$ chain decays. Although there are no published measurements of the neutron flux in the Davis laboratory, the environmental neutron back- 
ground can be estimated by scaling the combined spectra of fission and $(\alpha, n)$ neutrons and the flux measured in the Gran Sasso laboratory [28] to match the contamination levels found in the Homestake rock. Using the conservative limit that the Davis cavern is completely lined by rhyolite rock, the estimated incident neutron flux is $16.2 \times 10^{-9} \mathrm{n} \mathrm{cm}^{-2} \mathrm{~s}^{-1}$. There is no significant expected variation in the neutron yield from concrete, which was selected for its low radioactivity, and did not have significant thickness [31]. The fast neutron flux is efficiently moderated by the water shield, and the total integrated flux reduction due to water plus geometry is $6 \times 10^{-22}$ for $\mathrm{E}>1 \mathrm{keV}$. This corresponds to $10^{-16} \mathrm{n} \mathrm{yr}^{-1}$ incident on the detector.

\subsubsection{Muon-Induced Neutron Backgrounds}

Cosmic ray muons contribute to the NR background through the generation of neutrons both in the shield and surrounding rock. The resulting neutron flux has a significant high-energy component $(\mathrm{E}>10 \mathrm{MeV})$, reduced by only $\sim 3$ orders of magnitude in the water shield [1]. The choice of a deep site is essential in controlling this neutron background.

The Davis laboratory is located in the $4850 \mathrm{ft}$ level of the Homestake mine, with an effective depth of $4.3 \pm$ $0.2 \mathrm{~km}$.w.e. This depth corresponds to a muon flux of $(4.4 \pm 0.1) \times 10^{-9} \mathrm{~cm}^{-2} \mathrm{~s}^{-1}[32]$ and an average muon energy of $320 \mathrm{GeV}$ [33]. The muon-induced high-energy neutron flux from the rock at underground sites can be deduced from the measurements of the muon-induced neutron yield in liquid scintillators at several underground sites, combined with simulations of neutron production and propagation in various materials. The estimated total flux and energy distribution can be fitted with empirical depth-dependent functions [32]. The neutron flux from the rock at Homestake is calculated to be $(0.54 \pm 0.01) \times$ $10^{-9} \mathrm{n} \mathrm{cm}^{-2} \mathrm{~s}^{-1}$, where the quoted error reflects simulation statistics only and does not reflect the full uncertainty due to local variations in rock content. The energy distribution is estimated by using the function fit parameters for the Gran Sasso laboratory, which is the closest possible site in depth to the Homestake $4850 \mathrm{ft}$ level for which neutron flux from rock has been measured. The water shield reduces the integrated flux of high-energy rock neutrons at the cryostat to $1 \times 10^{-7} \mathrm{n} \mathrm{s}^{-1}$, resulting in a SS $\mathrm{NR}$ rate of $60 \mathrm{nDRU}_{n r}$ in the $3.4-25 \mathrm{keV}_{n r}$ range, for a $100 \mathrm{~kg}$ fiducial volume.

The muon-induced neutron production in the water shield can be estimated from the neutron yield in different materials calculated using FLUKA [32], and the event rate is determined by Monte Carlo simulations using GEANT4. For a water tank of $7.6 \mathrm{~m}$ diameter and $6.1 \mathrm{~m}$ height, the total neutron flux at the LUX outer cryostat is $6.3 \times 10^{-7} \mathrm{n} \mathrm{s}^{-1}$. The resulting NR event rate in the $100 \mathrm{~kg}$ fiducial region is $120 \mathrm{nDRU}_{n r}$ in the energy range of 3.4$25 \mathrm{keV}_{n r}$. The rate is only $\times 2$ larger than that from rock neutrons because water neutrons have less water shield to travel through to reach the detector, and thus have a lower average energy and make a smaller contribution to the neutron rate.

The total nuclear recoil background due to muon-induced high-energy neutrons, including the components from the cavern rock and generated in the water itself, is 0.1 WIMPlike NR events in the energy range of $3.4-25 \mathrm{keV}_{n r}$, in $100 \mathrm{~kg} \times$ one-year. The projected rate meets the external neutron background goal. External backgrounds are not considered for the 85.3 day WIMP search run, and are listed in the summary for the one-year run in Table 7.

\section{ER/NR Discrimination}

LUX relies on differences in the ionization to scintillation ratio between ER and NR events to provide rejection of low-energy ER events falling inside the WIMP search fiducial volume. The discrimination power is mapped through a combination of LUXSim/NEST Monte Carlo studies and direct calibration measurements.

The position and width of the ER S2/S1 band was mapped in the WIMP search range using $10^{4}{ }^{3} \mathrm{H}$ decays [2]. The ${ }^{3} \mathrm{H}$ was delivered into the active region by injection of tritiated methane, using a system developed by LUX collaborators [15]. The system will be described in detail in a future LUX publication [34]. Tritium offers a unique ability to map detector ER response in the WIMP search range, decaying by a naked $\beta$ with endpoint $18.6 \mathrm{keV} .45 \%$ of ${ }^{3} \mathrm{H}$ decays fall within the $0.9-5.3 \mathrm{keV}_{e e}$ LUX WIMP search energy range. The measured ER band $\pm 1.28 \sigma$ as a function of S1 photoelectrons is shown in Fig. 9a.

The NR band (also shown on the same figure) shape was generated using LUXSim NR events, convolved with both measured LUX S1 and S2 detection efficiencies and NEST photon and electron distributions. Direct NR calibration through $\mathrm{AmBe}$ and ${ }^{252} \mathrm{Cf}$ sources was used to verify the simulated NR band mean and width. These sources did not provide sufficient statistics for detailed NR band mapping, due to the large detector volume and the low fraction of SS NR events achievable with external calibration sources; however the results of the calibration showed statistical consistency with the model expectation. The NR calibration program will be described in detail in a future LUX publication [35].

It is worth noting that the very small amounts of methane introduced into the detector (ppt level or below) for this calibration fall well below the level at which one would worry about impacting light or charge yields. This was measured with concentrations of order percent, to be a factor 2 effect [36]. LUX further checked this by injecting ppm level of natural methane into the detector and monitoring the stability of the response to ${ }^{83 m} \mathrm{Kr}$ calibration lines.

The measured ER leakage fraction is shown in Fig. 9b. The ER leakage fraction is defined as the fraction of ER events falling below the NR band centroid in a given S1 
bin. Note that this ER discrimination definition corresponds to a "cut-and-count" WIMP search analysis, analogous to that performed for previous Xe dark matter experiments [37, 38]. LUX dark matter search results from the 85.3 day run used a profile likelihood ratio (PLR) analysis that extended the dark matter event search above the NR band centroid [2]. The ER leakage fraction is used as a conservative expectation of the ER background rejection power for the detector.

The leakage fraction is calculated both from direct measurement in ${ }^{3} \mathrm{H}$ calibration, and from a Gaussian fit to the ER band. The average leakage fraction in the WIMP search energy range 2-30 phe, for both direct measurement and Gaussian fits, is 0.04. This corresponds to $99.6 \% \mathrm{ER}$ discrimination.

\section{Comparison with Measured Low-Energy Data}

The predicted WIMP search ER background from the sources listed in Sec. 2 is compared with measured LUX background data from the 85.3 day WIMP search run. The WIMP search fiducial volume is used, defined as a cylinder with a radius of $18 \mathrm{~cm}$ and a height of $40 \mathrm{~cm}$. The fiducial volume is centered both radially and vertically in the detector, with $7 \mathrm{~cm} \mathrm{Xe}(1.4 \mathrm{~cm}$ in the drift region) below and $7 \mathrm{~cm}(6.5 \mathrm{~cm}$ in the drift region) above. Backgrounds are evaluated over the WIMP search energy range, 2-30 phe $\left(0.9-5.3 \mathrm{keV}_{e e}\right)$, in order to encompass the ${ }^{127} \mathrm{Xe}$ spectral shape. All other background spectra are flat in energy, and do not change the differential background measurement.

The radial, height and S1 distributions in the WIMP search fiducial volume for simulation and measured data are shown in Fig. 10. The S1 spectrum is constructed based on LUXSim background studies, NEST photon and electron yields, and measured LUX S1 and S2 detection efficiencies, using the same technique performed for construction of the NR band in Sec. 3. The measured and simulated background rates as a function of position in the detector are shown in Fig. 11.

Use of Kolmogorov-Smirnov (K-S) tests for the height and S1 distribution shapes yields p values of $26 \%$ and $94 \%$ respectively when testing the measured data against the simulated distributions. The radial distribution is measured to be systematically flatter than simulation predictions, with a K-S test $\mathrm{p}$ value of $0.004 \%$. The background expectation averaged over the entire fiducial volume and WIMP search run is given in Table 6 .

WIMP search ER backgrounds in the range $0.9-5.3 \mathrm{keV}_{e e}$ in the fiducial volume are shown after the June 14 midpoint of the WIMP search run in Fig. 12. The fiducial ER background rate in the first half of the run is $4.4 \pm$ $0.4_{\text {stat }} \mathrm{mDRU}_{e e}$, while the background rate in the second half is $2.8 \pm 0.4_{\text {stat }} \mathrm{mDRU}_{e e}$. The rate drop is a factor $\times 2.7$ higher than that predicted due to the decay of cosmogenic ${ }^{127} \mathrm{Xe}$ alone. However, the background rate in the second half of the run is consistent with the predicted $2.2 \pm 0.3 \mathrm{mDRU}_{e e}$ due to time-independent sources $\left({ }^{214} \mathrm{~Pb}\right.$,

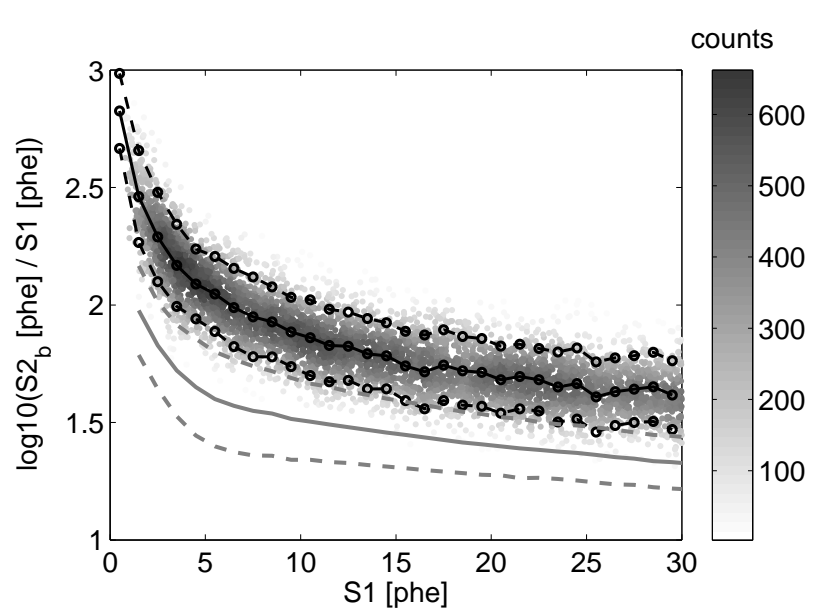

(a)

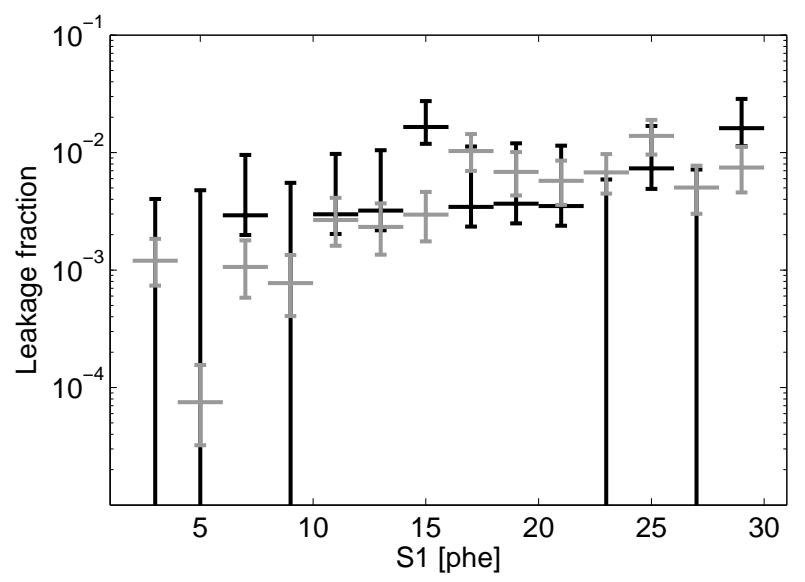

(b)

Figure 9: (a) ER S2/S1 band in LUX, as measured by ${ }^{3} \mathrm{H}$ calibration. The ${ }^{3} \mathrm{H}$ run yielded 4400 decays in the $118 \mathrm{~kg}$ fiducial volume, and are used to map the band mean and width. The measured band centroid is overlaid (solid), with $\pm 1.28 \sigma$ contours (dashed). The NR band, calculated using NEST and verified by neutron calibration, is shown in gray (red in color). (b) Measurement of ER band leakage below the NR band centroid for the LUX 85.3 day WIMP search run. Points are shown corresponding to the measured leakage fraction in each S1 bin (black), and the projected leakage fraction based on a Gaussian fit to the ER band in each bin (gray, red in color). Data is volume-averaged over the entire $118 \mathrm{~kg}$ fiducial volume. Measured points are taken from ${ }^{3} \mathrm{H}$ calibration. Errors shown are $\pm 34.1 \%$. 


\begin{tabular}{cc}
\hline Source & Background Rate $\left[\mathbf{m D R U}_{e e}\right]$ \\
\hline \hline$\gamma$ rays & $1.8 \pm 0.2_{\text {stat }} \pm 0.3_{\text {sys }}$ \\
${ }^{127} \mathrm{Xe}$ & $0.5 \pm 0.02_{\text {stat }} \pm 0.1_{\text {sys }}$ \\
${ }^{214} \mathrm{~Pb}$ & $0.11-0.22(0.20$ assumed $)$ \\
${ }^{85} \mathrm{Kr}$ & $0.17 \pm 0.10_{\text {sys }}$ \\
\hline \hline Total predicted & $2.6 \pm 0.2_{\text {stat }} \pm 0.4_{\text {sys }}$ \\
Total observed & $3.6 \pm 0.3_{\text {stat }}$ \\
\hline
\end{tabular}

Table 6: Predicted and measured low-energy background rates in the LUX $118 \mathrm{~kg}$ WIMP search fiducial during the 85.3 day run. Rates are averaged over the energy range $0.9-5.3 \mathrm{keV}_{e e}$.

${ }^{85} \mathrm{Kr}$, and $\gamma$ rays from construction materials), with an additional $0.28 \pm 0.06 \mathrm{mDRU}_{e e}$ from ${ }^{127} \mathrm{Xe}$. A K-S test yields a $\mathrm{p}$ value of $87 \%$ from comparison of the measured and simulated population distribution shapes.

The observed event distribution in $\mathrm{S} 2 / \mathrm{S} 1$ is consistent with an ER population, with total rate matching predictions based on modeling work [2]. From the measured $99.6 \%$ discrimination factor, an average 0.64 events are expected to fall below the NR band centroid. One event is observed at the NR centroid, at 3 phe S1. The PLR WIMP search analysis finds agreement with the background-only hypothesis with a $\mathrm{p}$ value of $35 \%$.

\section{Background Projections for the One Year Run}

The background studies from the 85.3 day WIMP search can be used to project the expected backgrounds for the 2014 one-year LUX WIMP search run. At the beginning of the one-year run, the ${ }^{127} \mathrm{Xe}$ background will have decayed below significance. The one-year run is also expected to use a more conservative $100 \mathrm{~kg}$ fiducial volume, further reducing position-dependent $\gamma$ ray backgrounds.

The predicted background sources within the $100 \mathrm{~kg}$ fiducial for the one-year run are listed in Table 7. A total of $1.4 \pm 0.2 \mathrm{mDRU}_{e e}$ is expected from all ER sources, assuming no change in ${ }^{214} \mathrm{~Pb}$ or ${ }^{85} \mathrm{Kr}$ rates from those observed in the 85.3 day run. The predicted total is in agreement with observations of data in a $100 \mathrm{~kg}$ fiducial during the second half of the 85.3 day run. The observed event rate is $1.7 \pm 0.3 \mathrm{mDRU}_{e e}$. The observed rate includes $0.15 \pm 0.04 \mathrm{mDRU}_{e e}$ of residual ${ }^{127} \mathrm{Xe}$, which will not be present during the one-year run. The neutron differential rate from both internal and external sources is $350 \mathrm{nDRU}_{n r}$.

Integrating over the $0.9-5.3 \mathrm{keV}_{e e}$ window for the ER sources and the equivalent $22 \mathrm{keV}_{n r}$ window for NR sources, and using the observed $1.7 \pm 0.3 \mathrm{mDRU}_{e e}$ and subtracting $0.15 \pm 0.04 \mathrm{mDRU}_{e e}{ }^{127} \mathrm{Xe}$, the total expected number of background events is $250(\mathrm{ER})+0.28(\mathrm{NR})$. After $99.6 \%$ ER discrimination, and assuming a $50 \%$ NR acceptance, the number of WIMP-like background events is $1.1 \pm 0.2$. The background rate is potentially further reduced by optimizing the shape of the fiducial volume to follow the

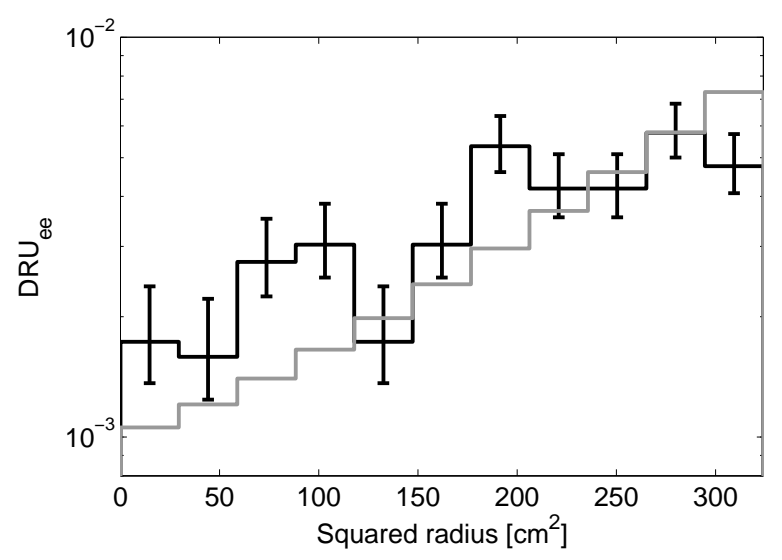

(a)

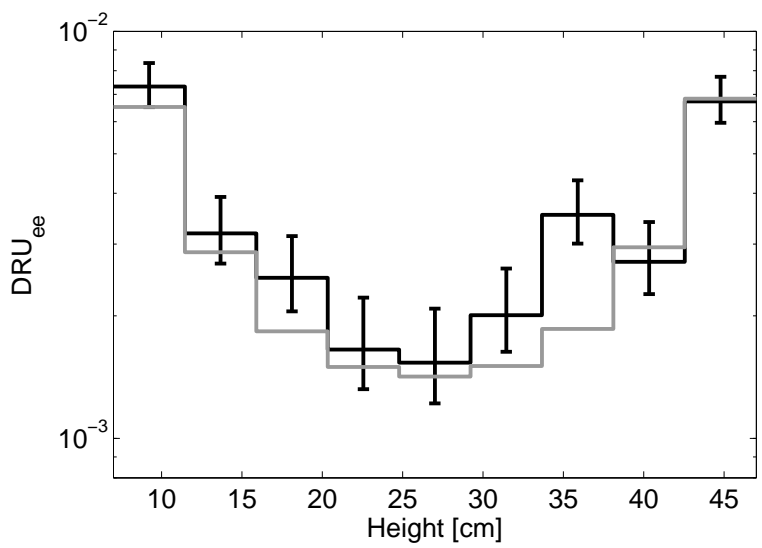

(b)

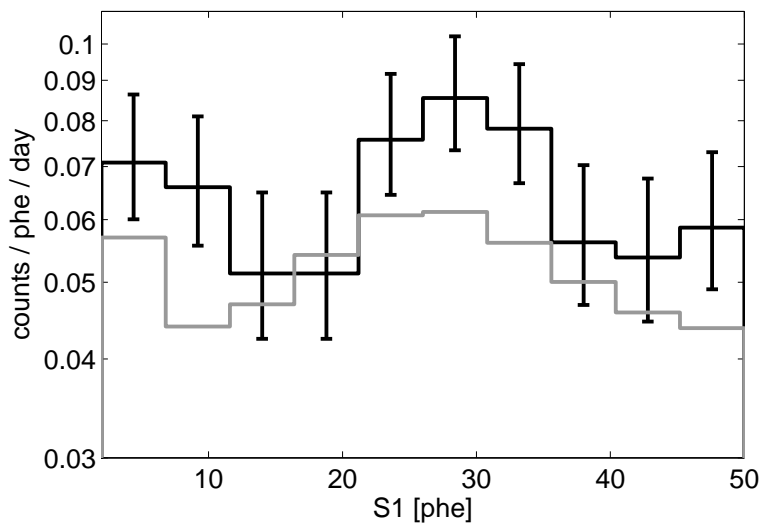

(c)

Figure 10: Low-energy measured distributions in (a) squared radius, (b) height, and (c) S1, within the LUX $118 \mathrm{~kg}$ fiducial volume, measured over the full 85.3 day WIMP search run. Measured data are indicated by the black histogram with error bars. Simulation data are shown as the gray histogram (red, in color). Simulated radial and height distributions are reconstructed from high-energy background studies, and are not a fit to low energy distributions. The energy spectrum predicted from $\gamma$ rays is normalized by the best-fit results in Table 3. The simulated S1 distribution folds in NEST estimates of total photon yields and measured LUX light collection efficiency. 


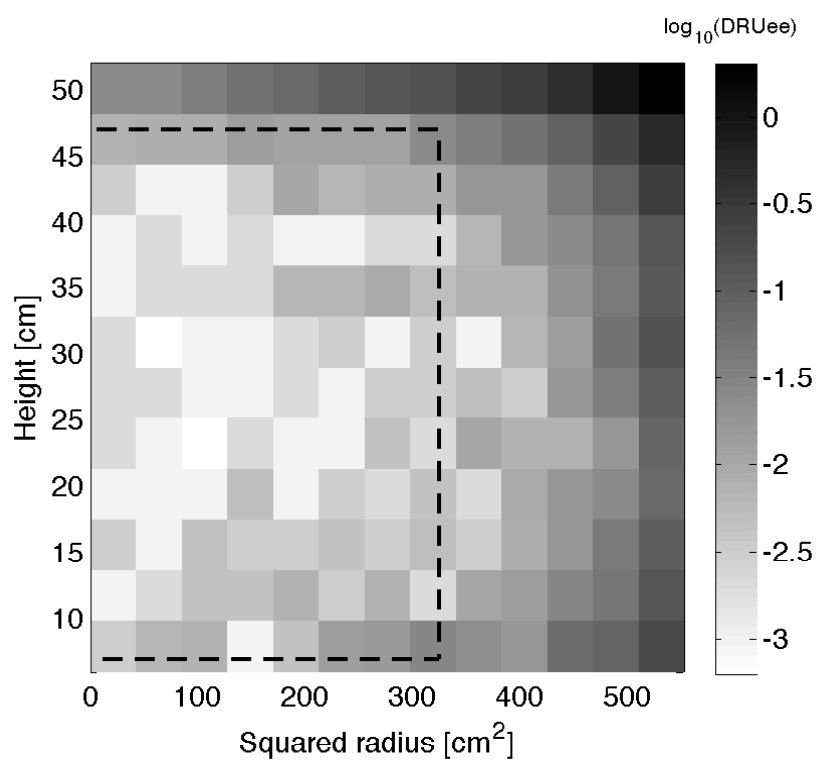

(a)

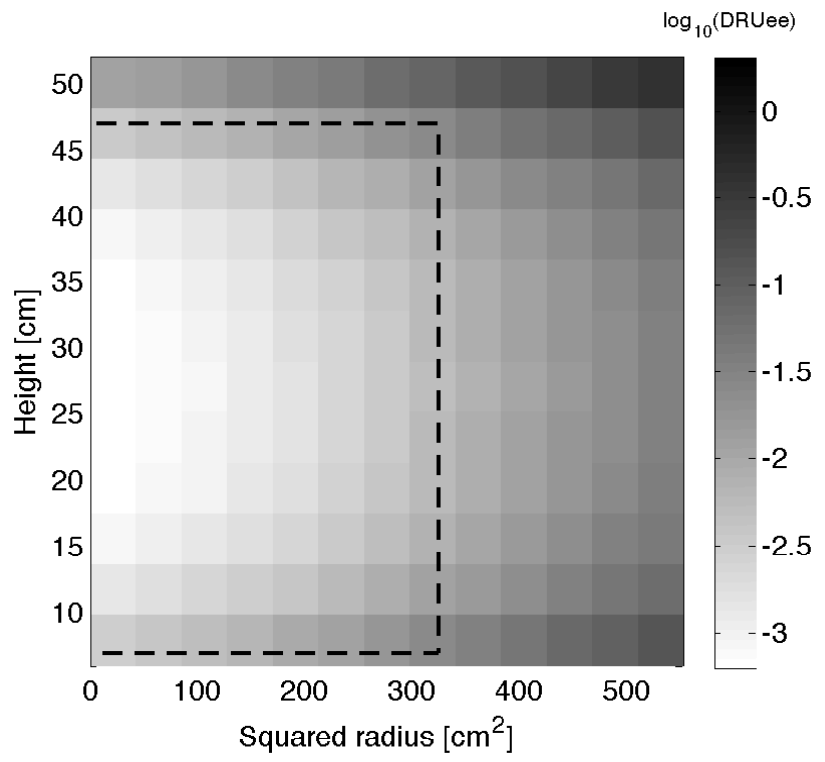

(b)

Figure 11: Low-energy background distributions in squared radius and height, from (a) measured data and (b) model predictions. Rates are taken in the range $0.9-5.3 \mathrm{keV}_{e e}(2-30 \mathrm{~S} 1 \mathrm{phe})$. Rates are shown in units of $\log _{10}\left(\mathrm{DRU}_{e e}\right)$. The $118 \mathrm{~kg}$ fiducial volume used in the 85.3 day WIMP search run is shown in dashed black. The model includes low-energy background contributions from $\gamma$ ray, ${ }^{127} \mathrm{Xe},{ }^{214} \mathrm{~Pb}$, and ${ }^{85} \mathrm{Kr}$ sources. Measured rates at large radii include a significant contribution from low-energy ${ }^{210} \mathrm{~Pb}$ decays at the detector walls. These decays are not included in the background model.

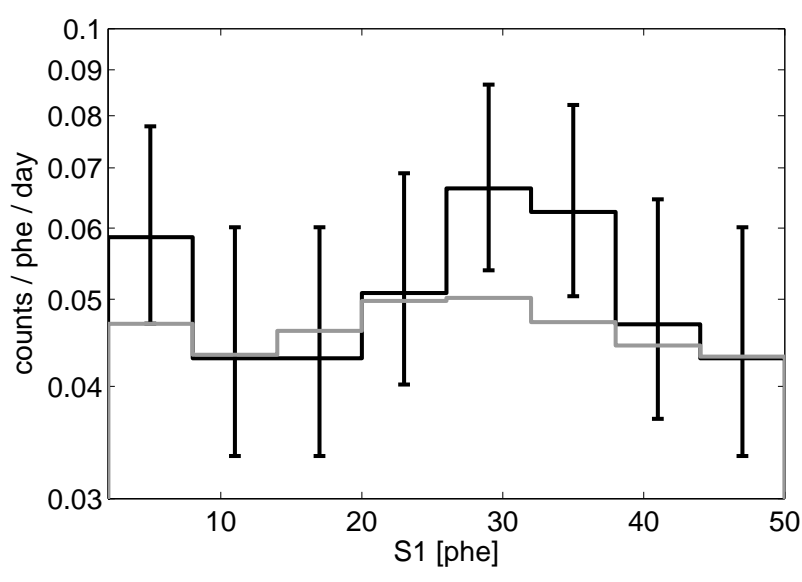

Figure 12: Low-energy ER backgrounds in the $118 \mathrm{~kg}$ fiducial volume during the second half of the 85.3 day WIMP search run (after June 14). Measured data are shown in black. The model predictions, including $\gamma$ ray, ${ }^{127} \mathrm{Xe},{ }^{214} \mathrm{~Pb}$, and ${ }^{85} \mathrm{Kr}$, are shown in gray (red, in color). Model S1 predictions are based on measured LUX efficiency factors and NEST photon and electron distributions. The measured data totals $2.8 \pm 0.4_{\text {stat }} \mathrm{mDRU}_{e e}$.

\begin{tabular}{cc}
\hline Source & Background Rate \\
\hline \hline$\gamma$ rays & $\left(1.0 \pm 0.1_{\text {stat }} \pm 0.1_{\text {sys }}\right) \mathrm{mDRU}_{e e}$ \\
${ }^{214} \mathrm{~Pb}$ & $0.2 \mathrm{mDRU}_{e e}$ \\
${ }^{85} \mathrm{Kr}$ & $\left(0.17 \pm 0.10_{\mathrm{sys}}\right) \mathrm{mDRU}_{e e}$ \\
Int. neutrons & $170 \mathrm{nDRU}_{n r}$ \\
Ext. neutrons & $180 \mathrm{nDRU}_{n r}$ \\
\hline \hline Total predicted & $1.4 \pm 0.2 \mathrm{mDRU}_{e e}+350 \mathrm{nDRU}_{n r}$ \\
\hline Total observed & $1.7 \pm 0.3 \mathrm{mDRU}_{e e}\left(0.14 \pm 0.03{ }^{127} \mathrm{Xe}\right)$ \\
\hline
\end{tabular}

Table 7: Predicted and measured low-energy background rates in a $100 \mathrm{~kg}$ WIMP search fiducial expected to be used for the one-year run. ER rates are averaged over the energy range $0.9-5.3 \mathrm{keV}_{e e}$. NR rates are averaged over the energy range $3.4-25 \mathrm{keV}_{n r}$. Measured rates are taken from the second half of the 85.3 day WIMP search run. The ${ }^{127} \mathrm{Xe}$ contribution to the observed background rate is given in brackets. This component will not be present during the one-year run.

background contours in the active region, and from optimizing the energy range for the observed background before the beginning of the run. The optimal shape will be determined by observed background rates before the start of the one-year run.

\section{Conclusions}

ER and NR low-energy backgrounds in the LUX experiment have been modeled in detail. Modeling work is based on Monte Carlo projections constrained by $\gamma$ ray assay of construction materials, as well as in-situ measurements of $\gamma$ rays and intrinsic radioisotope decay rates 
performed outside of the WIMP search fiducial volume and energy range. Low-energy background predictions are not directly fit from Monte Carlo but rather extrapolated from high-energy measurements. The use of independent measurements to set the model parameters and the resulting good agreement between low-energy projections and observed data gives high confidence that the low-energy backgrounds in LUX are well understood.

The primary backgrounds in the LUX detector arise from low-energy depositions from $\gamma$ ray scatters in the fiducial region. The $\gamma$-rays are generated from radioisotope decays in detector construction materials. The R8778 PMTs are the largest source of $\gamma$ ray backgrounds, with additional contributions from insulation materials. Cosmogenic production of ${ }^{60} \mathrm{Co}$ in $\mathrm{Cu}$ contributes a $\gamma$ ray rate $\times 3$ higher than expected based on initial screening results.

Measurements of $\alpha$ particle energy depositions in the detector provide a model for radon daughter decays in the fiducial volume. Alpha decay rates, combined with highenergy spectrum measurements, provide a constraint on ${ }^{214} \mathrm{~Pb}$ rates within a factor of $\times 2 .{ }^{85} \mathrm{Kr}$ backgrounds are calculated from direct measurements of nat $\mathrm{Kr}$ in LUX Xe.

The LUX 85.3 day WIMP search run background rate was elevated above expectations due to the presence of cosmogenically produced ${ }^{127} \mathrm{Xe}$. This isotope creates a low-energy ER background through the coincidence of lowenergy X-ray generation and high-energy $\gamma$ ray de-excitation, where the $\gamma$ ray escapes detection by leaving the active region. This isotope decays with a 36 day half-life, and contributes an extra $0.5 \mathrm{mDRU}_{e e}$ to the 85.3 day WIMP search run backgrounds. The backgrounds generated by this isotope will not be present in future dark matter search runs.

Neutron emission rates from $(\alpha, \mathrm{n})$ reactions, ${ }^{238} \mathrm{U}$ fission, and high-energy muon interactions are predicted to create a subdominant NR background in LUX. A search was performed for low-energy MS events in the detector, as such events would be a signature of neutron scattering. No NR-like MS events below the $50 \% \mathrm{NR}$ acceptance mean were found during the 85.3 day run, consistent with predicted neutron emission rates. Neutron scatter rates within the WIMP search fiducial and energy regions are projected to be comparable between internal and external sources.

The ER S2/S1 band was characterized by high-statistics ${ }^{3} \mathrm{H}$ calibration. The measured ER discrimination factor in LUX is $99.6 \%$, where NR events are characterized as falling below the NR S2/S1 band centroid.

Measured low-energy background rates are within $1 \sigma$ of expectation. An additional transient background during the first half of the WIMP search run was measured, in excess of expectations from ${ }^{127} \mathrm{Xe}$. The average background rate during the WIMP search run was $3.6 \pm 0.4 \mathrm{mDRU}_{e e}$. 0.64 events are projected to fall below the NR centroid in the 85.3 day WIMP search data set, based on measured ER rates. One event was observed at the NR centroid, with none falling below. The data taken during the 85.3 day run show an overall agreement with the background-only model, with a $\mathrm{p}$ value of $35 \%$.

The projected background rate for the 2014 one-year $\times 100 \mathrm{~kg}$ WIMP search run is $1.7 \pm 0.3 \mathrm{mDRU}_{e e}$, with a negligible NR background. The projected one-year run background rate is reduced by $55 \%$ relative to 85.3 day rate due to the decay of all transient backgrounds, as well as the use of a smaller fiducial volume. Further reductions in background are expected in particular from optimization of the shape of the fiducial volume to minimize positiondependent background contributions. The model predicts a strong WIMP discovery potential for LUX for the upcoming one-year WIMP search run.

\section{Acknowledgements}

This work was partially supported by the U.S. Department of Energy (DOE) under award numbers DE-FG0208ER41549, DE-FG02-91ER40688, DE-FG02-95ER40917, DE-FG02-91ER40674, DE-NA0000979, DE-FG02-11ER41738, DE-SC0006605, DE-AC02-05CH11231, DE-AC52-07NA27344, and DE-FG01-91ER40618; the U.S. National Science Foundation under award numbers PHYS-0750671, PHY-0801536, PHY-1004661, PHY-1102470, PHY-1003660, PHY-1312561, PHY-1347449; the Research Corporation grant RA0350; the Center for Ultra-low Background Experiments in the Dakotas (CUBED); and the South Dakota School of Mines and Technology (SDSMT). LIP-Coimbra acknowledges funding from Fundação para a Ciência e Tecnologia (FCT) through the project-grant CERN/FP/123610/2011. Imperial College and Brown University thank the UK Royal Society for travel funds under the International Exchange Scheme (IE120804). The UK groups acknowledge institutional support from Imperial College London, University College London and Edinburgh University, and from the Science \& Technology Facilities Council for Ph. D. studentship ST/K502042/1 (AB). The University of Edinburgh is a charitable body, registered in Scotland, with registration number SC005336. This research was conducted using computational resources and services at the Center for Computation and Visualization, Brown University.

We acknowledge the work of the following engineers who played important roles during the design, construction, commissioning, and operation phases of LUX: S. Dardin from Berkeley, B. Holbrook, R. Gerhard, and J. Thomson from University of California, Davis; and G. Mok, J. Bauer, and D. Carr from Lawrence Livermore National Laboratory. We gratefully acknowledge the logistical and technical support and access to laboratory infrastructure provided to us by the Sanford Underground Research Facility (SURF) and its personnel at Lead, South Dakota. SURF was developed by the South Dakota Science and Technology authority, with an important philanthropic donation from T. Denny Sanford, and is operated by Lawrence Berkeley National Laboratory for the Department of Energy, Office of High Energy Physics. 
[1] D. Akerib, et al., "The Large Underground Xenon (LUX) experiment," NIM A704 (2013) 111 - 126. [link].

[2] D. S. Akerib, et al., "First results from the LUX dark matter experiment at the Sanford Underground Research Facility,"ArXiv e-prints (Oct., 2013) . arXiv: 1310.8214 [astro-ph.CO].

[3] G. R. Blumenthal, et al., "Formation of galaxies and large-scale structure with cold dark matter,"Nature 311 (Oct., 1984) 517-525. [doi].

[4] M. Davis, et al., "The evolution of large-scale structure in a universe dominated by cold dark matter," $A p J 292$ (May, 1985) 371-394. [doi].

[5] D. Clowe, et al., "A Direct Empirical Proof of the Existence of Dark Matter," ApJ 648 (Sept., 2006) L109-L113. astro-ph/0608407.

[6] S. Agostinelli, et al., "Geant4-a simulation toolkit," Nuclear Instruments and Methods in Physics Research Section A: Accelerators, Spectrometers, Detectors and Associated Equipment 506 (2003) no. 3, 250 - 303. [link].

[7] D. Akerib, et al., "LUXSim: A component-centric approach to low-background simulations," NIM A675 (2012) 63 - 77 . [link].

[8] M. Szydagis, et al., "Enhancement of NEST capabilities for simulating low-energy recoils in liquid xenon," Journal of Instrumentation 8 (2013) no. 10, C10003. [link].

[9] D. Akerib, et al., "An Ultra-Low Background PMT for Liquid Xenon Detectors," NIM A703 (2013) 1-6. [link].

[10] D. S. Akerib, et al., "Radio-assay of Titanium samples for the LUX Experiment," ArXiv e-prints (2011) . arXiv:1112.1376 [physics.ins-det].

[11] D. C. Malling, et al., "Dark Matter Search Backgrounds from Primordial Radionuclide Chain Disequilibrium," ArXiv e-prints (May, 2013) . arXiv:1305.5183 [astro-ph.IM]

[12] J. J. Back and Y. A. Ramachers, "ACTIVIA: Calculation of Isotope Production Cross-sections and Yields," NIM A586 (2008) 286. arXiv:0709.3472.

[13] S. Cebrian, et al., "Cosmogenic activation in germanium and copper for rare event searches," Astroparticle Physics $\mathbf{3 3}$ (2010) 316 - 329. [link].

[14] M. Gordon, et al., "Measurement of the flux and energy spectrum of cosmic-ray induced neutrons on the ground,"Nuclear Science, IEEE Transactions on $\mathbf{5 1}$ (Dec., 2004) 3427-3434. [doi].

[15] A. Dobi, et al., "Study of a zirconium getter for purification of xenon gas," NIM A620 (2010) no. 2-3, 594 - 598. [link].

[16] D. Akerib, et al., "The \{LUX\} prototype detector: Heat exchanger development," Nuclear Instruments and Methods in Physics Research Section A: Accelerators, Spectrometers, Detectors and Associated Equipment 709 (2013) no. 0, 29 36. [link].

17] D. Akerib, et al., "Technical results from the surface run of the LUX dark matter experiment," Astroparticle Physics $\mathbf{4 5}$ (2013) 34 - 43. [link].

18] "Evaluated Nuclear Structure Data File," 2013. [link].

[19] J. E. Gaiser, Charmonium Spectroscopy from Radioactive Decays of the $J / \psi$ and $\psi^{\prime}$. PhD thesis, Stanford University, August, 1982.

20] V. V. Kuz'minov, et al., "Radioactive Kr85 in Krypton Enriched with a Light Isotope," Atomic Energy 73 (Dec., 1992) 1010-1011.

[21] A. I. Bolozdynya, et al., "A chromatographic system for removal of radioactive 85Kr from xenon,"NIM A579 (Aug., 2007) 50-53.

[22] D. S. Akerib, et al., "Chromatographic separation of radioactive noble isotopes from xenon for LUX experiment," In preparation (2014) .

[23] A. Dobi, et al., "Detection of krypton in xenon for dark matter applications," NIM A665 (2011) 1-6. arXiv:1103.2714 [astro-ph.IM] .

[24] D. Mei and C. Zhang, "Neutron Yield in Materials," 2008. [link]. http://neutronyield.usd.edu/.
[25] D. M. Mei, C. Zhang, and A. Hime, "Evaluation of (alpha,n) Induced Neutrons as a Background for Dark Matter Experiments," Nucl. Instrum. Meth. A606 (2009) 651-660. arXiv:0812.4307 [nucl-ex].

[26] L. de Viveiros, Optimization of Signal versus Background in Liquid Xe Detectors used for Dark Matter Direct Detection Experiments. PhD thesis, Brown University, May, 2010.

[27] A. Smith, "Homestake (DUSEL) Samples - results of Radiometric Analyses at LBNL," tech. rep., LBNL, 2007.

[28] H. Wulandari, et al., "Neutron flux at the Gran Sasso underground laboratory revisited," Astroparticle Physics 22 (Nov., 2004) 313-322. [link].

[29] NCRP, "NCRP Report No. 94: Exposure of the Population in the United States and Canada from Natural Background Radiation," Tech. Rep. 94, National Council on Radiation Protection \& Measurements (NCRP), 1988.

[30] M. Carson, et al., "Veto performance for large-scale xenon dark matter detectors," NIM A548 (2005) no. 3, 418 - 426. [link].

[31] J. Heise, "The Sanford Underground Research Facility at Homestake," Proceedings of the VII International Conference on Interconnections between Particle Physics and Cosmology (2014) .

[32] D. Mei and A. Hime, "Muon-induced background study for underground laboratories," Phys. Rev. D 73 (2006) 053004. arXiv: astro-ph/0512125.

[33] D. E. Groom, N. V. Mokhov, and S. I. Striganov, "Muon Stopping Power and Range Tables $10 \mathrm{MeV}-100 \mathrm{TeV}$," Atomic Data and Nuclear Data Tables 78 (July, 2001) 183-356. [doi].

[34] D. S. Akerib, et al., "Internal Calibration of the LUX Detector Using Tritiated Methane," In preparation (2014) .

[35] D. S. Akerib, et al. In preparation (2014)

[36] K. Pushkin, "Scintillation light, ionization yield, and scintillation decay times in high pressure xenon and xenon methane," IEEE Trans. Nucl. Sci. (2007) no. 54, 744-750.

[37] J. Angle, et al., "First Results from the XENON10 Dark Matter Experiment at the Gran Sasso National Laboratory," Phys. Rev. Lett. 100 (2008) 021303. [link].

[38] V. N. Lebedenko, et al., "Results from the first science run of the ZEPLIN-III dark matter search experiment,"Phys. Rev. D 80 (Sep, 2009) 052010. [link]. 